\title{
3D outer bulge structure from near infrared star counts ${ }^{\star}$
}

\author{
S. Picaud ${ }^{1,2}$ and A. C. Robin ${ }^{1}$ \\ 1 CNRS UMR6091, Observatoire de Besançon, BP 1615, 25010 Besançon Cedex, France \\ e-mail: [picaud; annie.robin] @obs-besancon.fr \\ 2 Astronomisches Rechen-Institut, Mönchhofstraße 12-14, 69120 Heidelberg, Germany \\ e-mail: picaud@ari.uni-heidelberg.de
}

Received 3 May 2004 / Accepted 13 June 2004

\begin{abstract}
We attempt to study the characteristics of the different stellar populations present in the Galactic central region. A Monte Carlo method is used to simultaneously fit 11 thin disc and triaxial outer bulge density parameters on $\left(K_{\mathrm{s}}, J-K_{\mathrm{s}}\right)$ star count data in almost 100 windows from the DENIS near infrared large scale survey at $-8^{\circ}<l<12^{\circ}$ and $|b|<4^{\circ}$. Various bulge density profiles and luminosity functions were tested using a population synthesis scheme. The best models, selected by a maximum likelihood test, give the following description: the outer bulge is boxy, prolate, and oriented $10.6^{\circ} \pm 3^{\circ}$ with respect to the Sun-center direction. It seems that the main bulge population is not older than $10 \mathrm{Gyr}$, but this preliminary result needs further work to be confirmed. A significant central hole is found in the middle of the thin disc. We discuss these results in regard to previous findings and the scenario of bulge formation.
\end{abstract}

Key words. Galaxy: structure - Galaxy: stellar content - Galaxy: bulge - Galaxy: disk

\section{Introduction}

Due to the high extinction as well as the superimposition of different stellar populations in the region, the structure of the inner Milky Way remains uncertain. In the last $10-15$ years, the use of infrared data, less sensitive to extinction, made it possible to make much progress in our knowledge of the bulge region. However, some questions such as the precise orientation and the length of the outer bulge, as well as the existence and the length of the central disc hole have received contradictory answers.

There is now a consensus that the outer bulge is triaxial: Blitz \& Spergel (1991) and Kent et al. (1991), using $2.4 \mu \mathrm{m}$ maps, and Nakada et al. (1991), using IRAS stars, detected asymmetries in longitude which they explained by the triaxiality of the outer bulge, also called the bar, with the near end at positive longitudes; Binney et al. (1991) studied the gas kinematics and came to the same conclusion. Then, studies using COBE/DIRBE maps (e.g. Dwek et al. 1995; Binney et al. 1997; Freudenreich 1998; Lépine \& Leroy 2000; Bissantz \& Gerhard 2002), star counts (e.g. Nikolaev \& Weinberg 1997; Stanek et al. 1997; López-Corredoira et al. 2000), kinematics (e.g. Deguchi et al. 2002; Zhao 1996) and microlensing (e.g. Zhao \& Mao 1996) made it possible to deduce a more detailed description of the triaxial bulge/bar, and in particular gave estimations of its orientation in the Galactic plane. But, if all studies converge to the description by a prolate shaped outer bulge with the major axis almost lying in the Galactic plane

* Tables 4-8 and Appendices A and B are only available in electronic form at http://www. edpsciences.org and the near end in the first quadrant, the estimated values of the angle of the major axis from the Sun direction vary between about $10^{\circ}$ and $30^{\circ}$. Some studies even present a bar with an angle around $40^{\circ}-45^{\circ}$ (e.g. Nakai 1992; Weinberg 1992; Deguchi et al. 1998; Sevenster et al. 1999; López-Corredoira et al. 2001). The reason for this difference is that they deal with two different things: López-Corredoira et al. (2001), then Picaud et al. (2003), showed the existence of a structure at $l=$ $20^{\circ}-27^{\circ}$, which may be at the top end of a long in-plane bar with an angle of about $40^{\circ}$ from the Sun-center direction. This structure is distinct from the triaxial outer bulge studied in the present work, but the confusion often appears in the literature.

Alard (2001) showed evidence for a nuclear bar in the most central parts of the Milky Way which may correspond to the inner bulge. But according to Ibata \& Gilmore (1995), the inner bulge and the outer bulge may be two distinct populations. Therefore, in order to avoid any confusion with another bar or with the inner bulge, we will give the name outer bulge to the triaxial prolate structure observed in the inner region $\left(|l| \leq 10^{\circ}\right)$ excluding the very central parts $\left(|l| \leq 1^{\circ}\right.$ and $\left.|b| \leq 1^{\circ}\right)$.

Another important stellar population present in the outer bulge region is the thin disc. The shape of the inner thin disc is still a controversial issue. In particular, the existence of a truncation or a hole at its center is still in doubt, even if there are more and more indications in this direction, in particular in external galaxies. For instance, Freeman (1970) classified the discs in two types, the first described by a single exponential, and the second modeled by the subtraction of two exponentials, i.e. with a central hole. Ohta et al. (1990), studying 6 early-type spiral galaxies, showed that all had Freeman 
type II discs. According to Bagget et al. (1996), the proportion of galaxies having an inner truncated disc is at least twice as numerous in barred spirals as in non-barred ones. In our Galaxy, Freudenreich (1998) fitted his bulge and disc model on the COBE/DIRBE map and found a disc hole radius of $3 \mathrm{kpc}$. More recently, Lépine \& Leroy (2000) showed that a disc model including the central hole was more convenient to fit the brigthness distribution and the rotation curve, and López-Corredoira et al. (2001) used a disc model with a truncation inside the Galactic ring at $3.7 \mathrm{kpc}$.

In this paper, we have compared simulated star counts from the Besançon model of the Galaxy with DENIS near infrared data in almost one hundred windows of low extinction distributed between $-12^{\circ}$ and $+8^{\circ}$ in longitude and $-4^{\circ}$ and $+4^{\circ}$ in latitude. Star counts are a better means than surface brightnesses to determine the 3-dimensional density parameters: integrated fluxes are dominated by the brightest and closest stars while star count studies take into account a wider range of intrinsic stellar luminosities and distances.

The article is organized as follows. In Sect. 2, we will describe the data (DENIS batches), the selection of low extinction windows and the selections made in color and magnitude. In Sect. 3, after a brief description of the Besançon model of the Galaxy, we will present the disc and outer bulge density distributions and luminosity functions (hereafter LF) used to compute the simulations and fit the parameters. In Sect. 4, we will describe the fitting method, based on Monte Carlo drawings and a maximum likelihood test. Results are given in Sect. 5 and compared with previous studies in Sect. 6. We conclude in Sect. 7.

\section{The data}

\subsection{DENIS batches}

The DENIS (Deep Near Infrared Survey of Southern Sky) survey (Epchtein et al. 1997; Fouqué et al. 2000) covers almost all the Southern Sky (97\% at the end of the survey) in strips of $30^{\circ} \times 12^{\prime}$. Photometric bands used are Gunn- $I(0.85 \mu \mathrm{m})$, $J(1.25 \mu \mathrm{m})$ and $K_{\mathrm{s}}(2.15 \mu \mathrm{m})$.

In addition to the survey strips, specific observations called "batches" (Simon et al., in preparation) were made in 1998 in outer bulge and plane regions in smaller fields (about $2 \mathrm{deg}^{2}$ ). Data reductions were made at PDAC (Paris Observatory Data Analysis Center). A PSF fitting optimised for the crowded fields was used for the source extraction. All standard stars observed in a given night were taken for the determination of the photometric zero point, resulting in accuracies ranging for 0.08 at $K_{\mathrm{s}}=8$ to 0.15 at $K_{\mathrm{s}}=13$, and 0.08 at $J=10$ to 0.15 at $J=15$.

\subsection{Windows of low extinction}

94 windows of about $15^{\prime} \times 15^{\prime}$ between the longitudes $\left[-12^{\circ}\right.$; $\left.8^{\circ}\right]$ and the latitudes $\left[-4^{\circ} ; 4^{\circ}\right]$ were selected in DENIS batches. These windows were selected from the Schultheis et al. (1999) extinction map as having either a low or homogeneous extinction which is easy to model. For most windows, the extinction distribution along the line of sight was modeled using no diffuse extinction but 2 clouds (localized extinction), one at $1 \mathrm{kpc}$ (Sagittarius-Carina arm) and the other at about $4 \mathrm{kpc}$ (Scutum-Centaurus arm). In a few windows, diffuse extinction was added. In each cloud, the $A_{\mathrm{V}}$ was estimated by comparing the quantiles of $J-K_{\mathrm{s}}$ color between data and simulations (see Sect. 3). The reddening in each photometric band was taken from the extinction law of Mathis (1990).

Unfortunately, as one can see in Fig. 1, there are almost no windows selected very close to the Galactic plane $\left(|b|<1^{\circ}\right)$, because of the large extinction there.

\subsection{Photometric bands and star selections}

In the present study, only magnitude $K_{\mathrm{s}}$ and color $J-K_{\mathrm{s}}$ were used to compare observed star counts with simulated ones, the $I$ band being too sensitive to extinction.

The blue side of the color-magnitude diagram (hereafter CMD) is mostly populated by foreground dwarfs, especially at faint magnitudes. Cuts were made in $K_{\mathrm{s}}$ and $J-K_{\mathrm{s}}$ to reduce this contamination: we kept only stars between 7.5 and an upper limit varying from field to field (12.5 on average) in $K_{\mathrm{s}}$, and with $J-K_{\mathrm{s}}>J-K_{\mathrm{s} / \mathrm{lim}}, J-K_{\mathrm{s} / \mathrm{lim}}$ being in the mean equal to 0.8 but also varying from field to field due to extinction. To ensure completeness, only stars below $\approx 15$ mag in $J$ have been kept.

\section{The model}

The Besançon model of the Galaxy has been used to compute simulations and compare them with the data. It is described in Sect. 3.1. The holed thin disc and triaxial outer bulge density models which have been fitted are presented there, as well as the luminosity function used.

\subsection{The Besançon model of the galaxy}

The Besançon model of stellar population synthesis aims at giving a global 3-dimensional description of the Milky Way structure and evolution, including stellar populations such as thin disc, outer bulge, thick disc and spheroid, as well as dark halo and interstellar matter. Details can be found in Robin \& Crézé (1986), Bienaymé et al. (1987) and Robin et al. (2003, 2004).

The approach of the Besançon model is semi-empirical: both theoretical schemes (stellar evolution, galactic evolution, galactic dynamics) and empirical constrained are used. Boltzmann and Poisson equations make it possible to self-consistently constrain the disc scale height via the Galactic potential. Simulated catalogues of stars are built and give observables (apparent magnitudes, colors, proper motion, radial velocities) directly comparable with the data. Interstellar extinction, photometric errors and Poisson noise are also added to make simulations as close as possible to observations.

The Besançon model has been used to determine the density laws and luminosity functions of the stellar populations: thin disc (Robin et al. 1992; Ruphy et al. 1996; Haywood et al. 1997), thick disc (Robin et al. 1996; Reylé \& Robin 2001), 


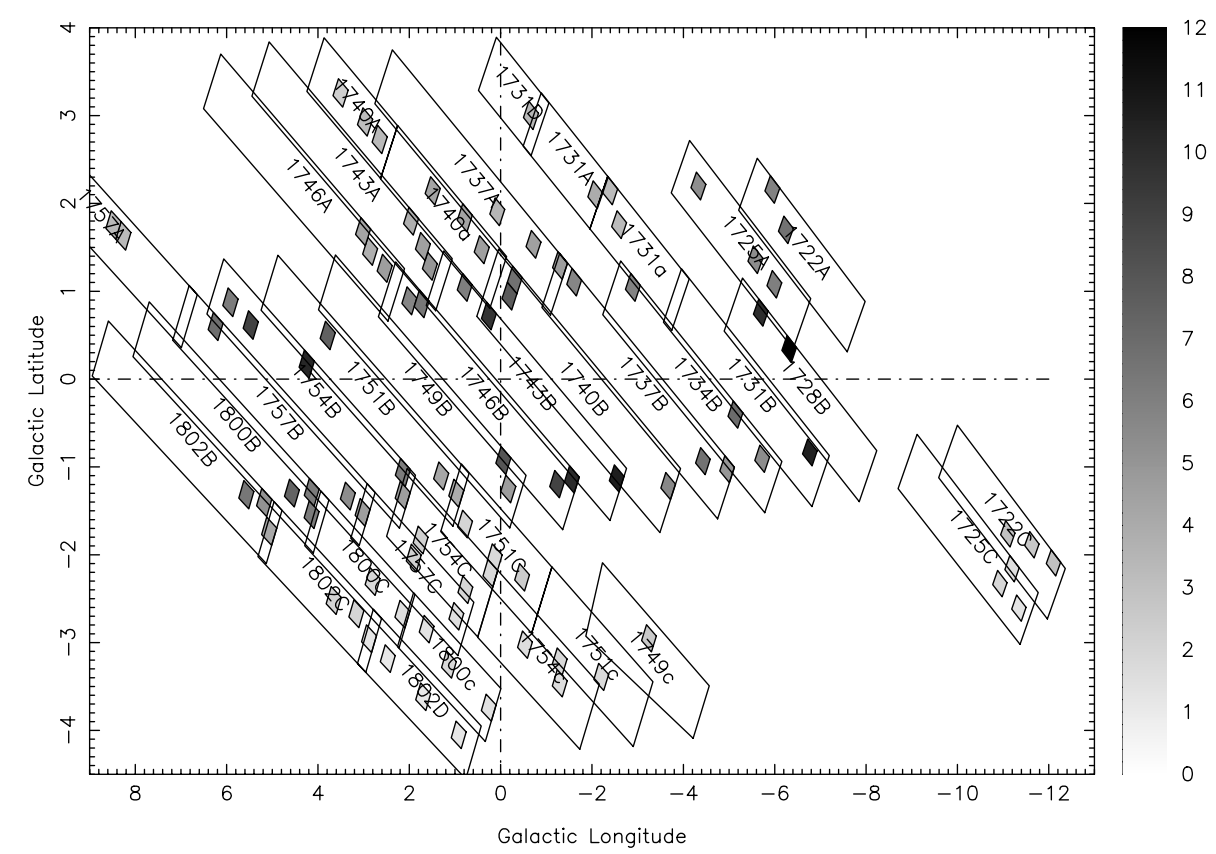

Fig. 1. Windows of low extinction (small filled boxes) and batches (long boxes). Grey levels of windows represent the $A_{\mathrm{V}}$ given by Schultheis et al. (1999).

spheroid (Robin et al. 2000), and outer bulge in the present work.

\subsection{Holed thin disc}

The thin disc may contribute significantly to star counts in the Galactic central region. Hence its characteristics have to be fitted at the same time as the bulge parameters. The two disc shape parameters that have an effect on star counts of the inner region are the scale lengths of the disc $\left(R_{\mathrm{d}}\right)$ and of its central hole $\left(R_{\mathrm{h}}\right)$. Other parameters and structures such as outer radius, warp and flare are taken into account in the Besançon model but are not relevant here.

\subsubsection{Density distribution}

The thin disc is divided into 7 age components: the first (age < $0.15 \mathrm{Gyr}$ ) is called young disc, the other six forming the old disc $(0.15 \mathrm{Gyr}<$ age $<10 \mathrm{Gyr})$. The young thin disc is not studied here, because its density is very low in comparison with the bulge and the old disc, and is probably very patchy. Fitting its parameters would be difficult and inefficient.

The old thin disc density distribution model follows the Einasto (1979) law: the distribution of each old disc component is described by an axisymmetric ellipsoid with an axis ratio depending on the age; the density law of the ellipsoid is described by the subtraction of 2 modified exponentials:

$\rho_{\mathrm{d}}=\rho_{\mathrm{d}_{0}} \times\left[\exp \left(-\sqrt{0.25+\left(\frac{a}{R_{\mathrm{d}}}\right)^{2}}\right)-\exp \left(-\sqrt{0.25+\left(\frac{a}{R_{\mathrm{h}}}\right)^{2}}\right)\right]$

with $a^{2}=R^{2}+\left(\frac{Z}{\epsilon}\right)^{2}$, where:

- $R$ and $Z$ are the cylindrical galactocentric coordinates;
Table 1. Axis ratios and local densities of the six age components of the old thin disc.

\begin{tabular}{ccc}
\hline \hline age $(\mathrm{Gyr})$ & $\epsilon$ & $\rho_{\mathrm{d}_{0}}\left(\star . \mathrm{pc}^{-3}\right)$ \\
\hline $0.15-1$ & 0.0268 & 0.03146 \\
$1-2$ & 0.0375 & 0.02538 \\
$2-3$ & 0.0551 & 0.01887 \\
$3-5$ & 0.0696 & 0.02625 \\
$5-7$ & 0.0785 & 0.02037 \\
$7-10$ & 0.0791 & 0.02284 \\
\hline
\end{tabular}

- $\epsilon$ is the axis ratio of the ellipsoid. Table 1 gives the recently revised (Robin et al. 2003) axis ratios of the 6 age components of the old thin disc;

- $R_{\mathrm{d}}$ is the scale length of the disc and is around $2.3-2.5 \mathrm{kpc}$ (Ruphy et al. 1996);

- $R_{\mathrm{h}}$ is the scale length of the hole;

- the normalization $\rho_{\mathrm{d}_{0}}$ is deduced from the local luminosity function (Jahreiß et al., private communication), assuming that the Sun is located at $R_{\odot}=8.5 \mathrm{kpc}$ and $Z_{\odot}=15 \mathrm{pc}$. Local densities are given in Table 1.

\subsubsection{Luminosity function}

A standard evolution model is used to produce the disc population, based on a set of evolutionary tracks, a constant Star Formation Rate (hereafter SFR) and a two-slope Initial Mass Function (hereafter IMF) $\phi(M)=A \cdot M^{-\alpha}$ with $\alpha=1.6$ for $M<1 M_{\odot}$ and $\alpha=3.0$ for $M>1 M_{\odot}$. The preliminary tuning of the disc evolution parameters against relevant 
Table 2. The 3 bulge density profiles used.

$\left.\begin{array}{cc}\hline \hline E & \rho_{E}=\rho_{0} \times \exp \left(-R_{s}\right) \\ G & \rho_{G}=\rho_{0} \times \exp \left(-0.5 \cdot R_{s}^{2}\right) \\ S & \rho_{S}=\rho_{0} \times \operatorname{sech}^{2}\left(-R_{s}\right)\end{array}\right\}$ with $R_{s}^{C_{\|}}=\left[\left|\frac{X}{x_{0}}\right|^{C_{\perp}}+\left|\frac{Y}{y_{0}}\right|^{C_{\perp}}\right]^{C_{\|} / C_{\perp}}+\left|\frac{Z}{z_{0}}\right|^{C_{\|}}$

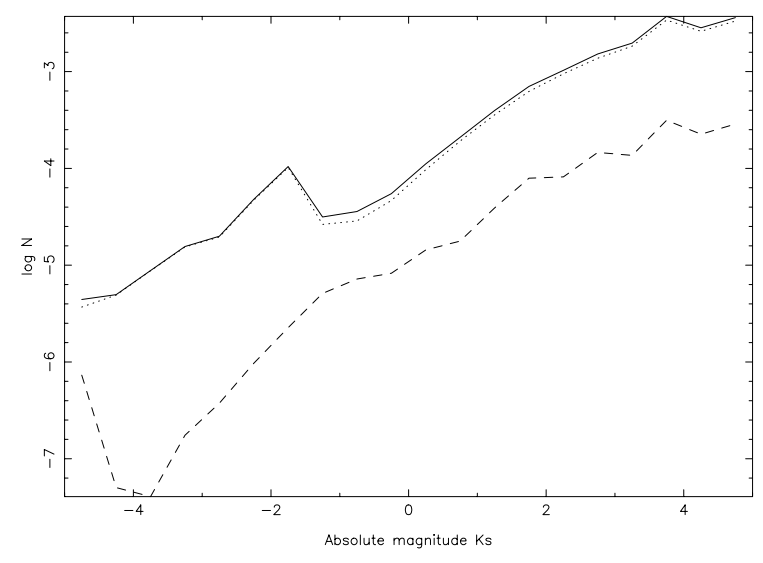

Fig. 2. Luminosity functions of the thin disc in the $K_{\mathrm{s}}$ band. The young disc corresponds to the dashed line, while the old one is represented by the dotted line. The solid line represents the total thin disc LF. On the ordinate: the decimal logarithms of the numbers of stars per $1 \mathrm{mag}$ bin of absolute magnitude.

observational data was described in Haywood et al. (1997) and further changes are explained in Robin et al. (2003).

Magnitudes and colors in various filters are computed using semi-empirical model atmospheres from Lejeune et al. (1997, 1998).

Figure 2 presents the luminosity functions in $K_{\mathrm{s}}$ of the young disc, the old disc and the thin disc in totality. This figure confirms how dominant the contribution of the old component on the thin disc is, in particular at the magnitudes $[-5 ;-2]$ which are the most frequent absolute magnitudes in the simulations of the present study. Within the range of apparent magnitude and color used in the present study, observed stars have absolute $K_{\mathrm{s}}$ magnitudes brighter than -1 .

\subsection{Triaxial bulge}

To simulate the star counts, the bulge density law and a luminosity function have to be assumed. The different bulge density profiles used are presented in Sect. 3.3.1.5 different luminosity functions have been tested, as explained in Sect. 3.3.2.

\subsubsection{Density distribution}

\section{Orientation}

There is consensus that the bulge is triaxial. Three angles define the orientation:

- $\phi$ : orientation angle from the sun-center direction of the projection on the Galactic plane of the bulge major axis;
- $\beta$ : pitch angle of the bulge major axis from the Galactic plane. In all previous studies, $\beta$ was found to be very close to $0^{\circ}$;

- $\gamma$ : roll angle around the bulge major axis.

However, the third angle $\gamma$ is ill defined because the minor axes have similar scale lengths. Hence we prefered to fix it at $\gamma=$ $0^{\circ}$, and only $\phi$ and $\beta$ have been fitted.

\section{Density profiles}

Dwek et al. (1995) and Freudenreich (1998) fitted various density distributions to the near infrared surface brightness observed using the Diffuse Infrared Background Experiment (DIRBE) of the Cosmic Background Explorer (COBE). The best-fitting models obtained at $2.2 \mu \mathrm{m}$ were the $G_{2}$ and $E_{1}$ functions by Dwek et al. (1995) and the $S$ function in Freudenreich (1998). Stanek et al. (1997) tested the same functions of Dwek et al. (1995) using star counts of red clump giants. Their bestfitting model, consistent with the observed star counts as well as the surface brightnesses used by Dwek et al. (1995), was the $E_{2}$ function.

We choose to test the 3 different functions used in these models, as presented in Table 2: an exponential function (called $E$ ) like $E_{1}$ and $E_{2}$, a Gaussian one (called $G$ ) like $G_{2}$, and the $S$ function of Freudenreich (1998), which is a sech ${ }^{2}$ function.

All the best density profiles obtained by Dwek et al. (1995), Stanek et al. (1997) and Freudenreich (1998) are included in the 3 functions described above: $E_{1}$ corresponds to the $E$ function with $C_{\|}=C_{\perp}=1$ (diamond shape), $E_{2}$ is obtained using the $E$ function and $C_{\|}=C_{\perp}=2$ (ellipsoidal shape), and the boxy profile $G_{2}$ using the $G$ function and $C_{\|}=4$ and $C_{\perp}=2$. The best values obtained in Freudenreich (1998), using the $\mathrm{S}$ function, are: $C_{\|}=3.501 \pm 0.0016$ and $C_{\perp}=1.574 \pm 0.0014$.

The density function is then multiplied by the cut-off function $f_{\mathrm{c}}$ (distances are given in $\mathrm{kpc}$, and $R_{\mathrm{c}}$ is called the cut-off radius):

$$
\begin{array}{ll}
\rho=\rho \times f_{\mathrm{c}}\left(R_{X Y}\right), & \text { with } R_{X Y}=\sqrt{X^{2}+Y^{2}} \\
R_{X Y} \leq R_{\mathrm{c}} \Longrightarrow & f_{\mathrm{c}}\left(R_{X Y}\right)=1 \\
R_{X Y} \geq R_{\mathrm{c}} \Longrightarrow & f_{\mathrm{c}}\left(R_{X Y}\right)=\exp \left(-\left(\frac{R_{X Y}-R_{\mathrm{c}}}{0.5}\right)^{2}\right) .
\end{array}
$$

Finally, the two angles $\phi$ and $\beta$, the three scale lengths $x_{0}, y_{0}$, $z_{0}$, the density at the center $\rho_{0}$, the cut-off radius $R_{\mathrm{c}}$ and the two coefficients $C_{\|}$and $C_{\perp}$ are considered as free density parameters in the fitting process.

\subsubsection{Luminosity functions}

Star counts are a function of both the density law and the luminosity functions. Five theoretical bulge luminosity functions 

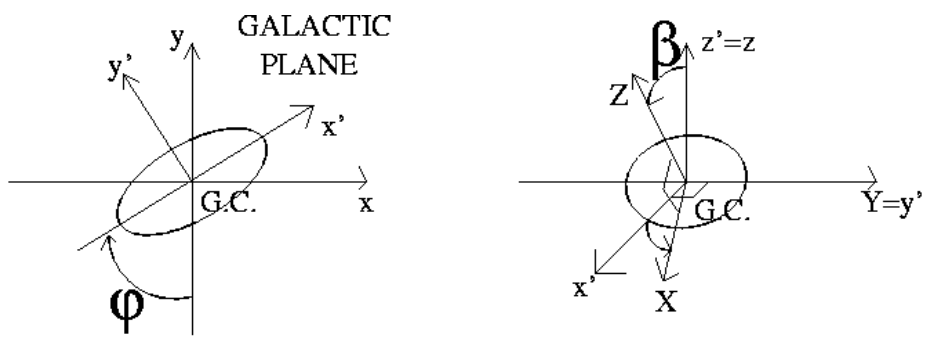

SUN

Fig. 3. Definition of the angles to pass from the Galactic frame $(x, y, z)$ to the bulge frame $(X, Y, Z)$. The transformation consists of 2 consecutive rotations: the first is a clockwise rotation of $\phi$ around the galactic vertical axis $z$, and the second is a clockwise rotation of $\beta$ around the new axis $y^{\prime}$.
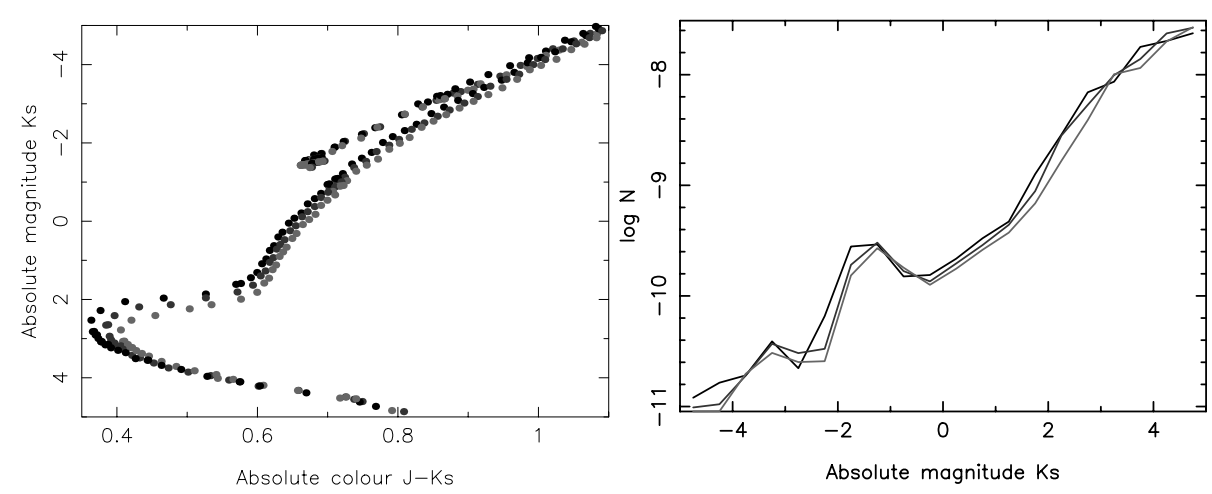

Fig. 4. Color-magnitude diagrams (left) and luminosity functions (right) from Padova models. The three models, Pad7.9, Pad10 and Pad12.6, are shown in the graphs using dark grey, grey and light gray respectively. The luminosity functions are given in number of stars per 0.5 mag bin of absolute magnitude $K_{\mathrm{s}}$.

have been tested, all of them based on a Salpeter IMF ( $\alpha=$ 2.35 ) and assuming a single epoch of formation (starburst) as well as a mean solar metallicity $(Z \approx 0.02)$. Only stellar evolutionary tracks and bulge age vary from one LF to another:

- Three of them (Fig. 4) have been deduced from theoretical isochrones by the Padova team (Girardi et al. 2002), and three bulge ages were tested: 7.9 Gyr, $10 \mathrm{Gyr}$ and 12.6 Gyr, which we shall name Pad7.8, Pad10 and Pad12.6 respectively. These luminosity functions were computed from the evolutionary tracks of Girardi et al. (2000) for the mass range $0.15 M_{\odot}-7.0 M_{\odot}$ and Bressan et al. (1993) for $M>$ 7.0 $M_{\odot}$, deduced from a combination of the Girardi et al. (2000) (with $Z=0.019$ and $Y=0.273$ ), and the Bertelli et al. (1994) $(Z=0.02, Y=0.280)$ non- $\alpha$-enhanced isochrones. Stellar atmosphere models were taken from the ATLAS9 theoretical spectra library (Castelli et al. 1997).

- The others two (Fig. 5) have been taken from evolutionary bulge synthesis models of Bruzual \& Charlot (see Bruzual et al. 1997). Models were constrained on several bulge globular clusters. Two bulge ages were tested: $10 \mathrm{Gyr}$ and 12 Gyr, respectively called BC10 and BC12. Evolutionary tracks were deduced from the Padova 1994 set (Alongi et al. 1993; Bressan et al. 1993; Fagotto et al. 1994a,b; Girardi et al. 1996), with $Z=0.02$ and $Y=0.280$. Stellar atmosphere models were taken from the semi-empirical Lejeune et al. $(1997,1998)$ spectral library.

While Bruzual \& Charlot luminosity functions are deduced from the Padova 1994 set of evolutionary tracks, those of
Girardi et al. (2002) use the Padova 2000 models, which are a new version of the Padova 1994 isochrones. The model atmospheres also differ: Girardi et al. (2002) use the theoretical spectra from Castelli et al. (1997), while Bruzual \& Charlot prefer the semi-empirical library from Lejeune et al. (1997, 1998).

Figure 6 compares Bruzual \& Charlot and Girardi et al. (2002) (Padova) models for an age of 10 Gyr. In Figs. 4-6, only dwarfs, subgiants, red giants, horizontal branch stars and AGB stars are shown. Planetary nebulae and white dwarfs, included in Bruzual \& Charlot models, are unobservable or negligible in star counts in the range of apparent magnitude used in the present work. However, they will be taken into account in the calculation of the total number of bulge stars in Sect. 5.5.

The LFs differ only slightly while CMDs are sensitive to the assumed age. The main age effect is the position of the turn-off, which corresponds to a color shift of about 0.05 mag for a change in age of 2 Gyr. The only non-negligible difference between Padova and Bruzual \& Charlot CMDs is that the Padova asymptotic giant branch coincides with the Bruzual red giant branch.

\section{Fitting method}

The method used to fit old thin disc and triaxial bulge parameters to DENIS data is based on two important points: firstly, comparisons between data and models are made with star counts in $K_{\mathrm{s}}$ magnitude and $J-K_{\mathrm{s}}$ color; secondly, 

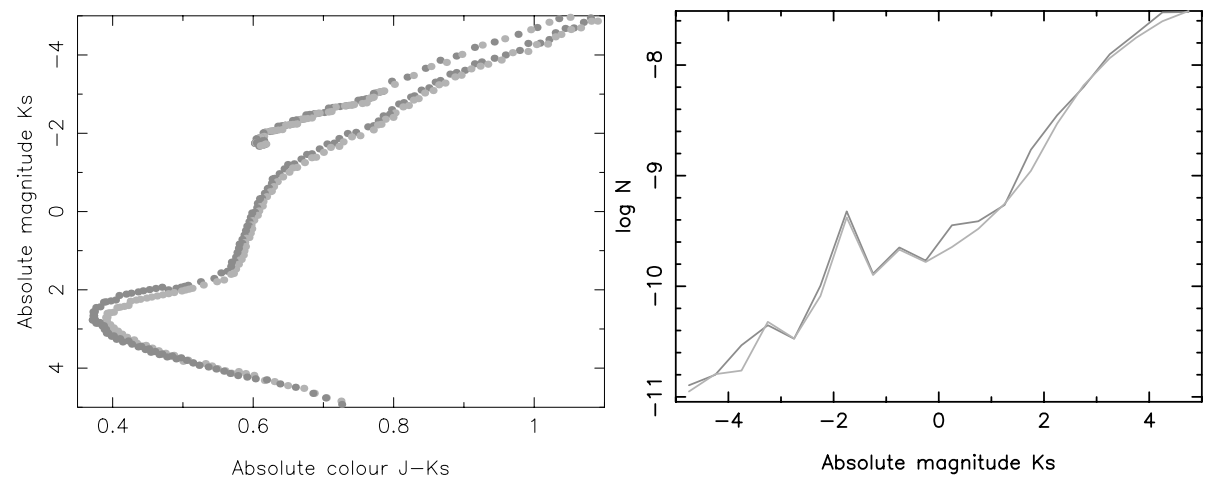

Fig. 5. Color-magnitude diagrams (left) and luminosity functions (right) from the Bruzual \& Charlot models. The darker grey corresponds to $\mathrm{BC} 10$ and the lighter grey to $\mathrm{BC} 12$. The luminosity functions are given in number of stars per 0.5 mag bin of absolute magnitude $K_{\mathrm{s}}$.
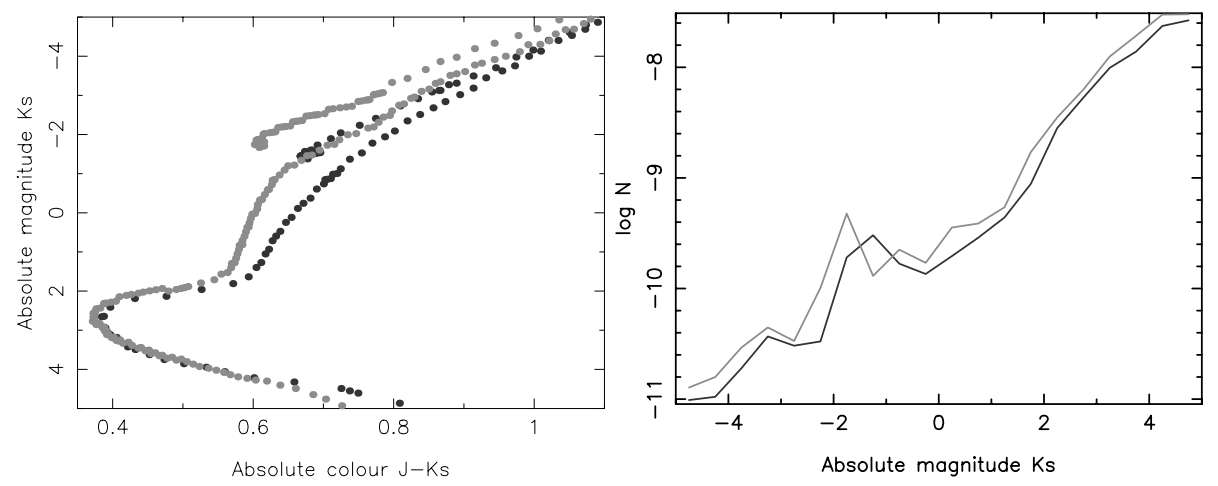

Fig. 6. Color-magnitude diagrams (left) and luminosity functions (right) from Padova (Pad10, dark grey) and Bruzual \& Charlot (BC10, light grey) for an age of $10 \mathrm{Gyr}$. The luminosity functions are given in number of stars per 0.5 mag bin of absolute magnitude $K_{\mathrm{s}}$.

parameters are deduced from a fitting method using Monte Carlo drawings and maximum likelihood tests.

\subsection{Cuts and choice of bin size}

For each field, selected stars are distributed in $8 \times 2$ equally populated bins: 8 bins of magnitude, and 2 bins of color. We noticed that the noise relative to the least populated bins increases and contributes too much to the global likelihood. This bias increases with the difference between data and model. To limit this bias, we group some close windows (always from the same batch) so that we have at least 70 stars in each bin of magnitude-color. Approximately $10 \%$ of the windows are grouped together by 2 or 3 , and we obtain 88 groups or single windows at the end, for 94 windows in total.

\subsection{Monte Carlo drawings}

There are 11 parameters to fit:

- Bulge orientation: $\phi, \beta$;

- Bulge scale lengths: $x_{0}$ (major axis), $y_{0}$ and $z_{0}$;

- Bulge normalization $\rho_{0}$ and cut-off radius $R_{\mathrm{c}}$;

- $C_{\|}$and $C_{\perp}$;

- Disc scale length $R_{\mathrm{d}}$ and hole scale length $R_{\mathrm{h}}$.

An iterative scanning method to explore this 11-dimensional space of parameters would be too time consuming. An alternative to save computer time would be to distribute the parameters in groups of 3 or 4 and fit them group by group. This method is still too slow and does not take into account correlations between parameters, and therefore can miss some solutions as well as preventing convergence in some cases. Hence we prefer to use another fitting method based on Monte Carlo drawings. This method was developed to solve non-linear equations of star kinematics (Oblak 1983) and has been adapted for the present study.

In the Monte Carlo method, parameters are drawn in the 11-dimensional parameter space.

- At the first iteration, $p$ points of the 11D space (or sets of parameters) are drawn using uniform drawings. Ranges for uniform drawings are given in Table 3;

- at each next iteration, the $m$ points having the highest values of likelihood among all the points drawn since the beginning are extracted. Then $p$ new points are determined using semi-Gaussian drawings around the median of the $m$ best points and along the axes defined by the eigenvectors of their correlation matrix. Formulae of median and semidispersions related to the semi-Gaussian drawings are given in Appendix A.

It can happen that drawn values go beyond restrictive limits. In this case, a new value is determined by a uniform drawing between the inferior/superior limit and the minimal/maximal value respectively reached by the $m$ best points on the concerned parameter. More details about the limits are given in Sect. 4.2.1; 
Table 3. Ranges for uniform drawings for the first iteration. Lower limits are given in the first line while upper limits are given in the second, and restrictions related to the limits applied in following iterations in the third. $\infty$ means no restriction, and [] corresponds to strict limits.

\begin{tabular}{|c|c|c|c|c|c|c|c|c|c|c|c|}
\hline Units & $\begin{array}{l}\phi \\
\circ\end{array}$ & $\begin{array}{l}\beta \\
\circ\end{array}$ & $\begin{array}{c}x_{0} \\
\mathrm{kpc}\end{array}$ & $\begin{array}{c}y_{0} \\
\mathrm{kpc}\end{array}$ & $\begin{array}{c}z_{0} \\
\mathrm{kpc}\end{array}$ & $\begin{array}{c}\rho_{0} \\
\star \cdot \mathrm{pc}^{-3}\end{array}$ & $\begin{array}{c}R_{\mathrm{c}} \\
\mathrm{kpc}\end{array}$ & $\begin{array}{c}R_{\mathrm{d}} \\
\mathrm{kpc}\end{array}$ & $\begin{array}{c}R_{\mathrm{h}} \\
\mathrm{kpc}\end{array}$ & $C_{\|}$ & $C_{\perp}$ \\
\hline Inf. & 0 & -10 & $0^{+}$ & $0^{+}$ & $0^{+}$ & $0^{+}$ & 1 & 2.2 & $0^{+}$ & 1 & 1 \\
\hline Sup. & 90 & 10 & 3 & 1 & 1 & 25 & 5 & 3 & 2.0 & 5 & 5 \\
\hline Restrictions & [] & $\infty$ & $>0$ & $>0$ & $>0$ & $>0$ & [] & [] & [] & [] & [] \\
\hline
\end{tabular}

- the fit ends when there is no further progress in the likelihood convergence. A maximum of 20 iterations is admitted, but this limit is almost never reached.

Various values of $m$ and $p$ have been tested and the values giving the best compromise between quality and rapidity of convergence were $m=40$ and $p=300$.

\subsubsection{Constraints on parameters}

Table 3 gives the ranges for the first iteration uniform drawings. These ranges were chosen to avoid unrealistic configurations, based on previous studies which agree on the following description: a prolate shaped bulge (small $y_{0}$ and $z_{0}$ with respect to $x_{0}$ ) with the major axis closer to the Galactic plane (small $\beta$ ) and its top end in the first quadrant $\left(0^{\circ} \leq \phi \leq 90^{\circ}\right)$. The following three points, concerning the limits, should be noted:

- in the case of the angle $\beta$, the limits apply during the first iteration only. Subsequent iterations can go beyond it;

- for certain parameters, such as the central density $\rho_{0}$ and the bulge scale lengths $x_{0}, y_{0}$ and $z_{0}$, only the inferior limit cannot be passed, and is equal to $0^{+}$;

- in the other cases (the parameters $C_{\|}$and $C_{\perp}$, the angle $\phi$, the cut-off radius $R_{\mathrm{c}}$ and the disc scale lengths $R_{\mathrm{d}}$ and $R_{\mathrm{h}}$ ), the limits are kept for all iterations.

\subsubsection{Maximum likelihood test}

Maximum likelihood methods are better than minimum $\chi^{2}$ ones, especially in the case of small counts per bin, because minimum $\chi^{2}$ methods assume that the distribution of observed star counts is a Gaussian, and this hypothesis is false in such a case. Hence, we preferred to use the maximum likehood to determine the best sets of parameters in the fitting method, even if normalized residuals and $\chi^{2}$ were also used as well as likelihood to evaluate, after the fits, the agreement between models and observations.

The use of the maximum likelihood method only assumes that the likelihood is smooth enough and well defined around its local maxima, which is eventually the case.

$\chi^{2}$ and likelihood formulae take into account the specificities of the study, especially the presence of noise in the simulations. They are presented in Appendix B.

\subsection{Weighting}

Computing simulations for each set of parameters drawn and for all windows would be very time consuming. Rather, we start with initial simulations, then weightings of these simulations are applied to adapt them to different density laws. Initial simulations were done using the $\mathrm{G}_{2}$ function $\left(C_{\|}=4\right.$, $C_{\perp}=2, \gamma=0^{\circ}$ ) from Dwek et al. (1995) as the bulge density distribution, densities with $E, G$ and $S$ distributions being calculated by changing the weightings adequately.

This method may lead to a bias if the values of parameters used for the initial simulations are badly chosen: if for instance the initial configuration implies that there are no bulge stars in a field, then there will be no bulge contribution for star counts in this field even for a drawn set of parameters which forecasts the presence of the bulge in the field. This bias is avoided by making simulations with appropriate values of parameters, i.e. no disc hole $\left(R_{\mathrm{h}}=0 \mathrm{kpc}\right)$, bulge scale-lengths all equal to the upper limit of the major scale-length $x_{0}(3 \mathrm{kpc})$, large bulge cut-off radius $(5 \mathrm{kpc})$.

A Poisson noise is added to the Besançon simulations to make them resemble the data as closely as possible. Initial simulations were computed with a density about 5 times greater to increase their signal to noise ratio.

\subsection{Convergence}

\subsubsection{Dispersions of best parameters}

Due to the Monte Carlo drawings, the $\mathrm{m}$ best sets of parameters of one fit are not exactly equal but their values of likelihood are very close, or even the same. Furthermore, two different fits do not give the same mean of best values. That is why 20 independent fits were made, the final result being the means and dispersions around the $20 \times m$ best values.

\subsubsection{Quality of convergence}

A convergence test was made using simulations to estimate the correlations and quality of convergence and to identify possible biases in the procedure. Three simulations were produced to be used as data and the full procedure were applied to them to attempt to retrieve the parameters used for these simulations. The three sets of parameters used to make these simulations have been chosen from randomly drawn sets to give three sufficiently different configurations for the thin disc and the outer bulge.

The main conclusions given by these three tests of convergence and correlations are the following:

- the values of the parameters are generally retrieved with good precision, around $6 \%$ of the range of the initial 


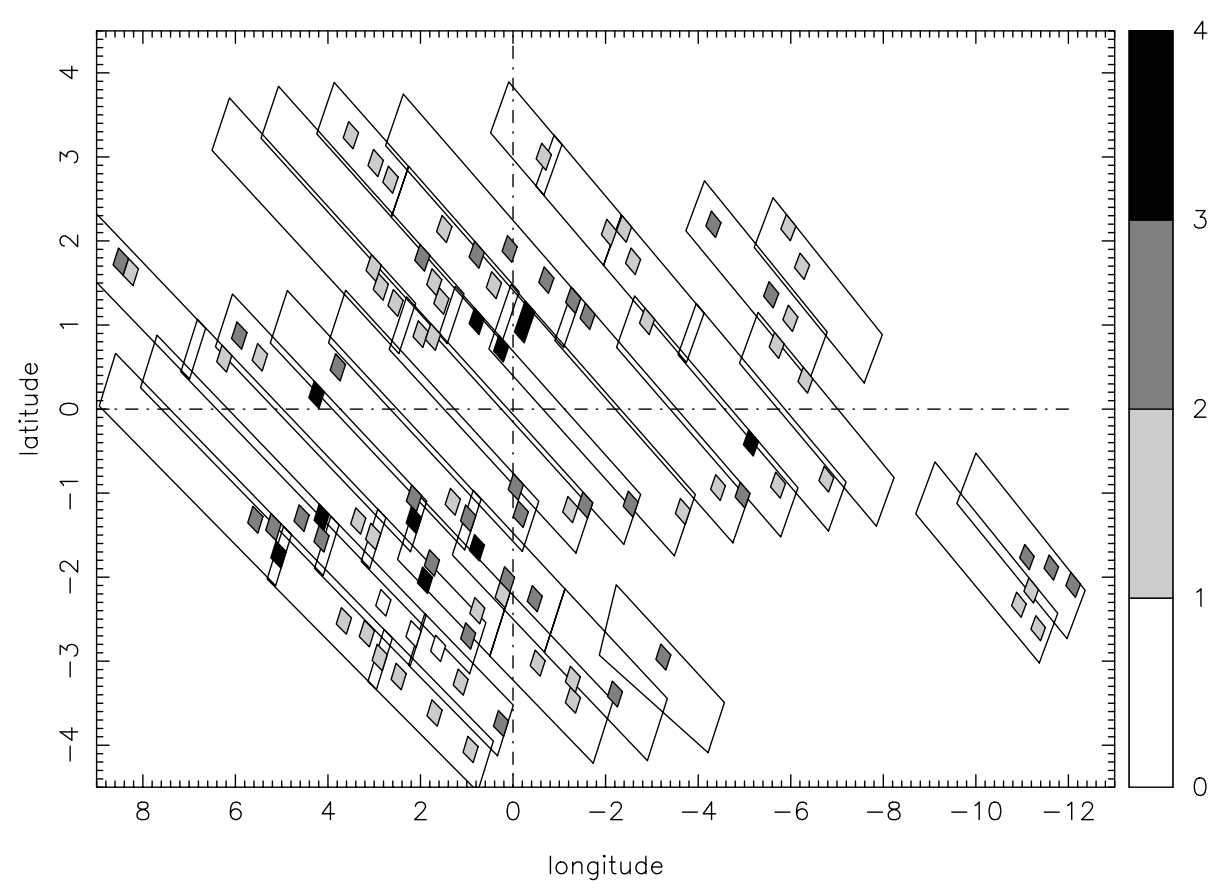

Fig. 7. Map of the mean $\chi_{r}$ (in number of $\sigma$ ) by window over the best sets of parameters obtained in the first round of fits using the Pad10 luminosity function. The maps using the other LF are almost identical.

drawings and $6 \%$ of the value, except for some parameters mentioned in the following lines;

- the accuracy is good: of the 33 values of parameters to be found, a third are retrieved at less than 0.5 sigmas, about $60 \%$ at less than 1 sigma, about $80 \%$ at less than $1.5,88 \%$ at $\leq 2$ and $97 \%$ at $\leq 2.5$;

- disc scale lengths $R_{\mathrm{d}}$ and $R_{\mathrm{h}}$ are strongly anticorrelated, which will be taken into account in the discussion. The disc parameters do not show any significant correlation with the bulge ones. However, $R_{\mathrm{d}}$ and $R_{\mathrm{h}}$ best values are sometimes a little biased with respect to the real values (varying from 0 to $2.4 \sigma$ difference);

- $C_{\|}$and $C_{\perp}$ are slightly anti-correlated to $y_{0}$ and $z_{0}$ respectively. Moreover, $C_{\|}$and $C_{\perp}$ are not precisely determined, having a dispersion between 0.5 and 0.7 , and a difference with the original values of up to 1.6 sigma;

$-\beta$ as well as the latitudes of the fields studied here being small, the line of sight is almost parallel to the plane, which implies a slight anti-correlation between $x_{0}$ and $\phi$. Furthermore, $\phi$ presents a slight correlation with the density at the center $\rho_{0}$. Moreover, the precision of $\phi$ depends on its value: it is good when $\phi=14^{\circ}$, less good when $\phi$ is small $\left(8.5^{\circ}\right)$ and much worse when $\phi$ is high $\left(37^{\circ}\right)$;

- the cut-off radius $R_{\mathrm{c}}$ is badly constrained, with dispersions varying between 0.3 and $0.85 \mathrm{kpc}$.

\section{Results of fits}

\subsection{First round}

The first round of fits presents a great degeneracy: the angle $\phi$ is found to converge towards two distinct solutions. While many fits converge to a median value in the range $5^{\circ}, 12.5^{\circ}$, many others give a value very close to $0^{\circ}$, which is inevitably biased because of the strict limit at $0^{\circ}$ for this parameter. Medians and dispersions of the 2 groups of solutions are tabled. The cut between the two partitions has been fixed at $\phi=3.5^{\circ}$.

The degeneracy does not depend on the tested density profile, but is more pronounced with Padova luminosity functions (especially for age $12.6 \mathrm{Gyr}$ ) than with Bruzual \& Charlot LFs.

\subsubsection{Identification of badly fitted windows}

Figure 7 presents the map of the mean $\chi_{r}$ (square root of $\chi^{2}$ per bin; see Appendix B.2 for more explanations) by window associated to the best parameters for the Padova luminosity function at $10 \mathrm{Gyr}$. The maps related to the different LF are similar, and the badly fitted windows are mostly the same, even if the $\chi_{r}$ values are a little smaller for the Bruzual \& Charlot luminosity functions. Mean $\chi_{r}$ are calculated over all the fits without taking into account the degeneracy.

Two groups of bad fields can be extracted:

- the first group of 8 windows is located at positive longitudes $\left(3^{\circ} \leq l \leq 6^{\circ}\right)$ and negative latitudes $\left(-2^{\circ} \leq b \leq\right.$ $0.5^{\circ}$ ), which contributes around $25 \%$ of the global likelihood. All these fields present a significant excess compared to model counts. A probable explanation is the following: these windows are amongst the most extincted of all the studied fields. Firstly, this implies that a poor modeling of the extinction distribution, fitted using the Bruzual \& Charlot BC10 luminosity function, has a larger effect on the star counts than expected. Secondly, because of this high extinction, the observed stars are intrinsically brighter than in other directions, and the poor agreement may be caused by a problem in the model for this range of absolute 
magnitudes, which has almost no effect on the star counts in other locations;

- a second group of 10 windows, located around the galactic center, contributes around $25 \%$ of the global likelihood. All these fields present a large deficiency in the star count compared to the model. This must be due to the absence of a stellar population in the Besançon model of the Galaxy, the inner bulge, distinct from the outer bulge that we are studying here, and confined to the first 1 or 2 degrees around the Galactic center. The presence of such a central population is mentioned by Ibata \& Gilmore (1995) and Frogel et al. (1999). The nuclear bar evoked by Alard (2001) could correspond to the same population.

These two different groups of badly fitted windows represent less than $20 \%$ of the total number of fields but contribute more than half of the global likelihood. Furthermore, the degeneracy in $\phi$ is mostly implied by them. As the aim of this study is to obtain a large scale description of the outer bulge and thin disc populations, we prefer to remove these fields and make new fits without them.

\subsection{Second round}

Tables 4 to 8 show the best parameters related to the second round fits for the 5 luminosity functions. For a given LF and a given density profile, the medians and dispersions around these medians of the $20 \times m$ best values of parameters have been calculated.

One can see that the likelihood has decreased strongly. The degeneracy in $\phi$ has almost disappeared with the Bruzual \& Charlot $\mathrm{BC} 10$ and $\mathrm{BC} 12$ and Padova Pad7.9 functions, and with the Padova Pad10 LF when the S density profile is used. It is less important but still present for the Padova Pad10 (using $\mathrm{E}$ and $\mathrm{G}$ luminosity functions) and Pad12.6 functions. As the fits converge away from $0^{\circ}$, the limit between the two groups of the degeneracy has been moved from $\phi=3.5^{\circ}$ to $\phi=4.5^{\circ}$.

The second group of fits (with $\phi<4.5^{\circ}$ ), which are much less numerous in the second round, is now considered an artefact, and only the other group of fits will be taken into account.

\subsection{Density profiles and luminosity functions}

Some conclusions can be deduced from Tables 4 to 8:

- Density profiles: the Gaussian function $(G)$ (the least peaked at the center) is the worst of the three bulge density profiles tested. $E$ and $S$ functions reach similar conclusions but give slightly different best values, especially for $\phi$ and its correlated parameters. The best agreement is obtained with the $S$ profile.

- Luminosity functions: at similar ages, the Bruzual \& Charlot luminosity functions give better agreement than the Padova (Girardi et al. 2002) ones. However, the best LF used come from Padova models with a bulge age of 7.9 Gyr. The Bruzual \& Charlot isochrone at a similar age was not available. This is the youngest tested value, which may mean that the bulge age might be smaller.
- Influence of the LF and density profile on best parameters: the thin disc scale length $R_{\mathrm{d}}$ and $R_{\mathrm{h}}$ determinations are robust over the different luminosity functions and density profiles which have been tested. This is not the same for the bulge parameters. For instance, the orientation angle $\phi$ is different with the $E$ profile and with the two others, and shows a significant dependency on the luminosity function: it is larger when the Bruzual \& Charlot LFs are used, and decreases when the age increases. Correlated parameters such as $R_{\mathrm{c}}$ or $x_{0}$ are also affected.

\subsection{Quality of fits and correlations}

Figure 8 shows the map of $\chi_{r}$ (square root of the $\chi^{2}$ per bin) associated to the fits using the Padova luminosity functions at 10 Gyr. The other maps related to other LFs are similar. One can see that the agreement is good (less than 3 sigmas) for all but one or two windows.

\subsubsection{Accuracy of the results}

The dispersions obtained for the majority of parameters are small, however some of them are not well constrained, such as the bulge shape coefficients $C_{\perp}$ and $C_{\|}$and the cut-off radius $R_{\mathrm{c}}$. Nevertheless, this is not a serious problem. A small variation of these parameters does not change significantly the bulge spatial distribution: $C_{\|}$and $C_{\perp}$ give only a general indication of the shape of the outer bulge, and do not have significant influence on other parameters, except a little on the bulge minor scale lengths $y_{0}$ and $z_{0}$ with which they are correlated. As for the cut-off radius, the scale length $x_{0}$ being short, the density varies by $10 \%$ to $20 \%$ at the distance $R_{\mathrm{c}}$, and the effect of the cut-off is not very strong.

\subsubsection{Correlations}

Table 9 shows the matrix of correlations around the best parameters for the second round of fits (group with $\phi>4.5^{\circ}$ only). The choice of the used luminosity function and density profile does not have any significant influence on the correlations, the matrix corresponds to the mean values over all the (LF, profile) pairs. For a given LF and a given density profile, correlations are computed, using all the fits, as follows: let $\left(\xi^{k}\right)$ be the $m \times 20$ best points and $w_{k}$ their weight (deduced from their likelihood); let us take two axis $i$ and $j$, the coordinates $\xi_{i}$ and $\xi_{j}$ of the points on these two axes, their weighted means $m_{i}=\sum_{k} w_{k} \cdot \xi_{i}^{k}$ and $m_{j}=\sum_{k} w_{k} \cdot \xi_{j}^{k}$, and their weighted dispersions $s_{i}=\sum_{k} w_{k} \cdot\left(\xi_{i}^{k}-m_{i}\right)^{2}$ and $s_{j}=\sum_{k} w_{k} \cdot\left(\xi_{j}^{k}-m_{j}\right)^{2}$; then the correlation between the parameters $i$ and $j$ is equal to $\frac{\sum_{k} w_{k} \cdot\left(\xi_{i}^{k}-m_{i}\right)^{2}><\left(\xi_{j}^{k}-m_{j}\right)^{2}}{s_{i} s_{j}}$.

The main conclusions given by these correlations are the following:

- the disc scale lengths $R_{\mathrm{d}}$ and $R_{\mathrm{h}}$ are not correlated with bulge parameters but are strongly anticorrelated: the variation in the density due to decreasing one of the two parameters can be compensated by increasing the other one. 


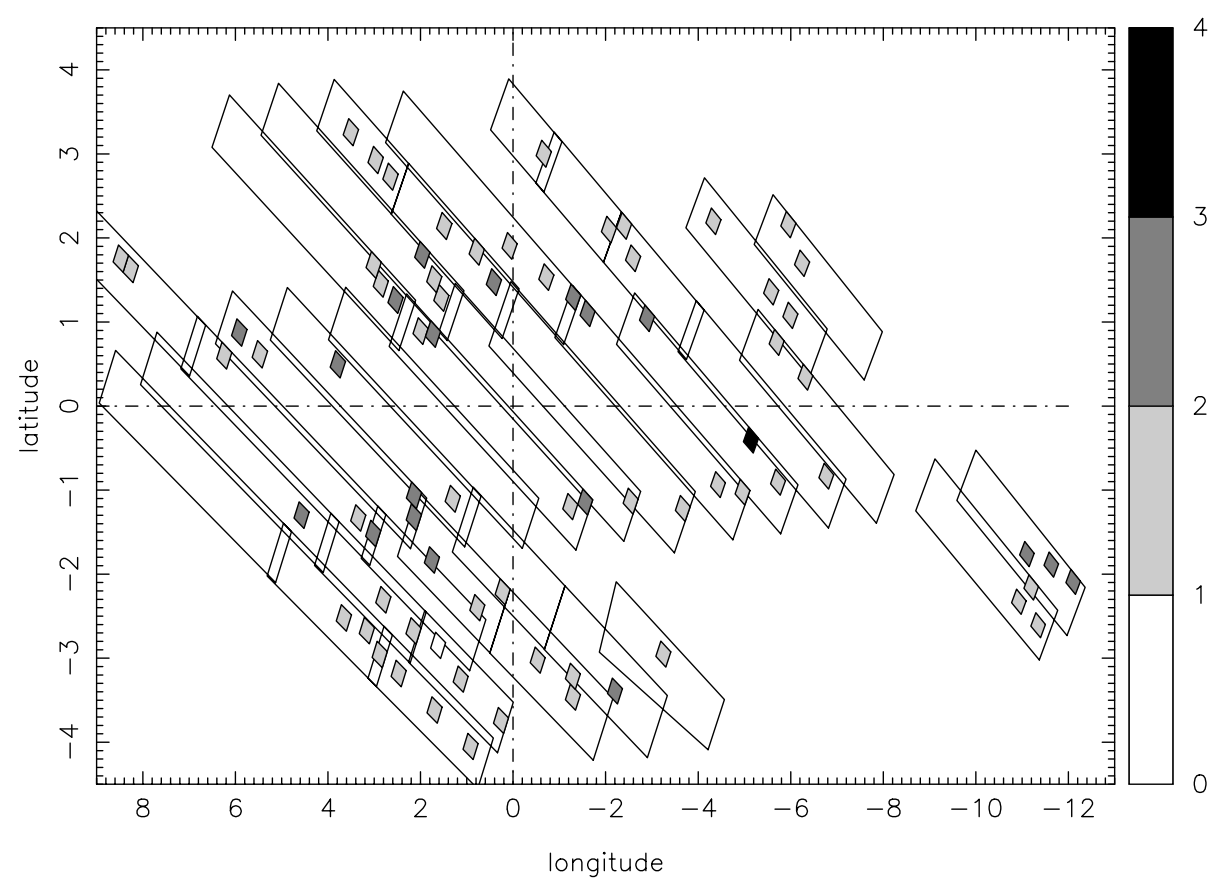

Fig. 8. Map of the mean $\chi_{r}$ by window related to the Pad10 luminosity function.

Table 9. Mean values over the 5 luminosity functions and the 3 density profiles of the correlations of the first group fits of the second round. Values over \pm 0.7 are written in boldface.

\begin{tabular}{ccccccccccc}
\hline \hline & $\beta$ & $x_{0}$ & $y_{0}$ & $z_{0}$ & $\rho_{0}$ & $R_{\mathrm{c}}$ & $R_{\mathrm{d}}$ & $R_{\mathrm{h}}$ & $C_{\|}$ & $C_{\perp}$ \\
\hline$\phi$ & 0.0 & $-\mathbf{0 . 7}$ & $\mathbf{- 0 . 7}$ & -0.3 & 0.6 & 0.2 & -0.2 & 0.0 & 0.5 & 0.6 \\
$\beta$ & & 0.1 & -0.1 & -0.4 & 0.0 & 0.1 & -0.1 & 0.3 & 0.1 & -0.1 \\
$x_{0}$ & & & 0.6 & 0.3 & $\mathbf{- 0 . 9}$ & -0.4 & 0.1 & 0.1 & -0.4 & -0.2 \\
$y_{0}$ & & & & 0.6 & -0.5 & -0.5 & 0.2 & 0.0 & -0.5 & -0.5 \\
$z_{0}$ & & & & -0.3 & -0.3 & 0.2 & -0.2 & -0.6 & -0.2 \\
$\rho_{0}$ & & & & & 0.3 & -0.1 & 0.0 & 0.2 & 0.1 \\
$R_{\mathrm{c}}$ & & & & & & & -0.1 & 0.1 & 0.1 & 0.1 \\
$R_{\mathrm{d}}$ & & & & & & & $-\mathbf{0 . 8}$ & -0.3 & -0.2 \\
$R_{\mathrm{h}}$ & & & & & & & & 0.0 & 0.1 \\
$C_{\|}$ & & & & & & & & & 0.3 \\
\hline
\end{tabular}

This results in an incertainty of the hole scale length $R_{\mathrm{h}}$, though without questioning the existence of the hole;

- as we have already shown with the tests (see Sect. 4.4.2), the orientation angle $\phi$ is anti-correlated with parameters such as $x_{0}$ and $y_{0}$;

- the bulge major scale length $x_{0}$ and central density $\rho_{0}$, whose variations have opposite effects on the number of stars at a given distance, are also strongly anti-correlated.

\subsection{Best parameters}

Table 10 gives values of the fitted parameters for the best model (using the Freudenreich $1998 \mathrm{sech}^{2}$ density profile and the Girardi et al. 2002 (Padova) luminosity function with an age of $7.9 \mathrm{Gyr}$ ), as well as the ones obtained by calculating the mean of the best values over all the pairs of density profile/luminosity function. These two sets of parameters are consistent with each other. We can therefore deduce that the configuration described here is robust, and does not depend on the choice of the LF and the density profile.

This configuration is the following:

- Outer bulge: the outer bulge is prolate, with axis ratios 1:0.31 \pm 0.4:0.26 \pm 0.3 (values from mean best models), and seems to be boxy in all directions $\left(C_{\perp}>2, C_{\|}>2\right)$. Its major axis lies almost in the Galactic plane $(\beta$ is consistent with the value $0^{\circ}$ ) and is oriented about $10^{\circ}$ with respect to the Sun-center direction. It contains $82 \times 10^{9} \pm$ $20 \times 10^{9}$ stars, which corresponds to a mass of $2.4 \pm 0.6 \times$ $10^{10} M_{\odot}$.

- Thin disc: the thin disc shows a large hole at its center. With a disc scale length of about $2.4 \mathrm{kpc}$ and a hole scale length of about $1.3 \mathrm{kpc}$, the in-plane density goes from zero at the center to its maximum at a distance of $2 \mathrm{kpc}$, and then decreases until the cut-off at $14 \mathrm{kpc}$. However, the behaviour of the disc in the inner $100 \mathrm{pc}$ is not constrained here. 
Table 10. Best values obtained from the second round of fits. The first double line corresponds to the model giving the best agreement with data, i.e. with the $S$ density profile and the Pad7.9 luminosity function. The values of the second one are the mean and dispersions of the best set of parameters obtained with each pair density profile/luminosity function. Meaning of the parameters: $\phi$ gives the orientation of the bulge major axis with respect to the Sun-center direction; $\beta$ is the angle between this bulge major axis and the Galactic plane; the bulge major scale length $x_{0}$, whose significance depends on the density profile, has been replaced by $\hat{x}_{0}$, which is the distance on the major axis at which the density is equal to $38.6 \%$ of the central one; $r_{y}=\frac{y_{0}}{x_{0}}$ and $r_{z}=\frac{z_{0}}{x_{0}}$ are respectively the axis ratios associated to the bulge minor scale lengths $y_{0}$ and $z_{0} ; N_{\text {tot }}$ gives the total number of bulge stars; $R_{C}$ is the cut-off radius of the outer bulge, and $C_{\|}$and $C_{\perp}$ correspond to its shape coefficients; $R_{\mathrm{d}}$ and $R_{\mathrm{h}}$ are respectively the scale lengths of the thin disc and of its central hole.

\begin{tabular}{cccccccccccccc}
\hline \hline & $\begin{array}{c}\phi \\
0\end{array}$ & $\begin{array}{c}\beta \\
\circ\end{array}$ & $\begin{array}{c}\hat{x}_{0} \\
\mathrm{kpc}\end{array}$ & $r_{y}$ & $r_{z}$ & $\begin{array}{c}N_{\mathrm{tot}} \\
10^{10} \star\end{array}$ & $\begin{array}{c}R_{\mathrm{c}} \\
\mathrm{kpc}\end{array}$ & $C_{\|}$ & $\begin{array}{c}C_{\perp} \\
R_{\mathrm{d}} \\
\mathrm{kpc}\end{array}$ & $\begin{array}{c}R_{\mathrm{h}} \\
\mathrm{kpc}\end{array}$ \\
\hline Best & $\mu$ & 10.6 & 0.8 & 1.97 & 0.30 & 0.25 & 6.39 & 3.71 & 3.38 & 3.49 & 2.35 & 1.31 \\
Model & $\sigma$ & 3.0 & 0.9 & 0.17 & 0.02 & 0.01 & 1.9 & 0.71 & 0.09 & 0.09 & 0.67 & 1.03 \\
\hline Mean best & $\mu$ & 9.4 & 0.6 & 1.74 & 0.31 & 0.26 & 8.24 & 3.25 & 3.40 & 3.68 & 2.40 & 1.26 \\
Models & $\sigma$ & 2.8 & 0.5 & 0.24 & 0.04 & 0.03 & 1.35 & 0.64 & 0.40 & 0.47 & 0.06 & 0.06 \\
\hline
\end{tabular}

\section{Discussion}

Many studies have been made of the structure of the outer bulge region. Here, we compare the description obtained by our fits with those found in the literature.

\subsection{Disc hole}

The strong anti-correlation between the two disc parameters $R_{\mathrm{d}}$ and $R_{\mathrm{h}}$ forces us to be careful with the results, which may be biased. Nevertheless, the hole radius value (assumed to be the radius of the maximal disc density), $\approx 2 \mathrm{kpc}$, is large enough to be used as evidence of the presence of a central hole in the inner thin disc.

This conclusion is in agreement with the studies of external galaxies by Ohta et al. (1990) and Bagget et al. (1996), who argued for the existence of holed or inner truncated discs in most spiral barred galaxies, as well as the Galactic disc models of Freudenreich (1998) who studied the CORBE/DIRBE map and obtained a hole radius of $3 \mathrm{kpc}$, López-Corredoira et al. (2001) who analysed DENIS data and found a maximum disc density at about $2 \mathrm{kpc}$ from the Galactic center, and Lépine \& Leroy (2000) who found that a model including a central hole is in better agreement with the COBE/DIRBE surface brightness distribution and the rotation curve.

However, a central hole or an inner truncation are not the only things which can give a smaller disc density in the plane close to the center. An alternative explanation is a vertical flare in the inner disc. The scale height of the disc may be higher there as an effect of the bar potential. Therefore, assuming that the surface density of the disc is constant, this flare leads to a decreasing of the disc density in and close to the Galactic plane, as does a central hole. For instance, López-Corredoira et al. (2004) found similar agreements with their data in the plane in the range $2-8 \mathrm{kpc}$ using either a flaring disc or a holed one. As a flaring disc model does not change the global density, contrary to a holed disc model, a further study of the vertical distribution of the inner thin disc might make it possible to settle between these two alternative models.

\subsection{Outer bulge age}

All the tested bulge luminosity functions have been built using the same scheme: the bulge is composed of only one generation of stars, which are rather old, and the mean metallicity has been assumed solar but with a large dispersion of 0.5 dex. These hypotheses are consistent with most constraints found in the literature.

The best agreement in our fits is obtained with the luminosity function from Girardi et al. (2002) with an age of 7.9 Gyr. This is the youngest tested age. Therefore, we can assume that the bulge age is at least younger than $10 \mathrm{Gyr}$, and perhaps even younger than $8 \mathrm{Gyr}$, which is in contradiction with the results of Zoccali et al. (2003) who propose $10 \mathrm{Gyr}$ as a minimum, but is in favour of Cole \& Weinberg (2002) who claimed that the outer bulge is not older than 6 Gyr. New luminosity functions with a younger age from Bruzual \& Charlot (2003) as well as from Girardi et al. (2002) (Padova) are now available and we shall attempt to use them in the future to test the hypothesis of a younger age, or several mixed generations of stars, as well as the influence of the assumed bulge metallicity.

\subsection{Outer bulge shape}

Our best density profiles are the $\operatorname{sech}^{2}$ function from Freudenreich (1998) and the exponential one proposed by Stanek et al. (1997), with a preference for the $\operatorname{sech}^{2}$ profile. On the contrary, the Gaussian function, the best model found by Dwek et al. (1995), gives the worst agreements.

The outer bulge as described by our best parameters is very boxy. This is consistent with most other works on the subject, for instance Weiland et al. (1994) and later articles (Dwek et al. 1995; Freudenreich 1998; ...) from the same authors, which studied integrated luminosities from the COBE/DIRBE near infrared map. Moreover, the axis ratios, 1:0.30 \pm 0.02:0.25 \pm 0.01 , which show that the triaxial bulge is very prolate, are similar to values obtained by Freudenreich (1998) (1:0.37:0.26), Dwek et al. (1995), Bissantz \& Gerhard (2002) (1:0.3-0.4:0.3, also from the COBE/DIRBE map) and Weiner \& Sellwood (1999) (1:0.33:0.33, using HI and CO data). This description 
of the outer bulge as a boxy prolate spheroid makes it similar to a bar.

Concerning its half-length, the cut-off radius $R_{\mathrm{c}}$ is fitted to $3.71 \pm 0.71 \mathrm{kpc}$. Using $x_{0}=1.82 \mathrm{kpc}$, assuming no cut-off, the bulge density on the major axis is equal to $3 \%$ of the central density at $4.4 \mathrm{kpc}\left(R_{\mathrm{c}}+1 \sigma\right)$ from the center, and $14 \%$ at $3 \mathrm{kpc}$ $\left(R_{\mathrm{c}}-1 \sigma\right)$. This means that a cut-off at $3.71 \mathrm{kpc}$ is too far to be very pronounced. This explains the small precision in $R_{\mathrm{c}}$. The existence of such a cut-off may be related to the disc dynamics. A cutoff may appear at the corotation associated with the spiral arms or the molecular ring. However, the position found here is a bit too far out and inaccurate to allow us to conclude that it is a dynamical cut-off.

\subsection{Outer bulge orientation}

As in most of the other related works, the angle $\beta$ found with our fits is very small and consistent with $0^{\circ}$, which means that the outer bulge major axis almost lies in the Galactic plane.

On the contrary, our estimation of $\phi$ (i.e. the angle between the bulge major axis and the Sun-center direction), $10.6^{\circ} \pm 3^{\circ}$, differs from some previous works. These studies, based on COBE/DIRBE surface brightness (Dwek et al. 1995; Binney et al. 1997; Bissantz \& Gerhard 2002), kinematics studies (Feast \& Whitelock 2000; Bissantz et al. 2002), or IRAS (Nikolaev \& Weinberg 1997; Deguchi et al. 2002) and OGLE star counts analyses (Stanek et al. 1997), give values around $20^{\circ}$. However our estimation is consistent with the $12^{\circ} \pm 6^{\circ}$ obtained by López-Corredoira et al. (2000), who analysed star counts from near infrared large scale survey as we did, and compatible with the $14^{\circ}$ found by Freudenreich (1998) and Lépine \& Leroy (2000), both using the COBE/DIRBE map, or even the $16^{\circ} \pm 2^{\circ}$ obtained by Binney et al. (1991) from gas kinematics.

It is not clear why different studies coming from the same data (COBE/DIRBE) give somewhat different results, which vary between $\phi=20^{\circ}-30^{\circ}$ and $\phi=14^{\circ}-16^{\circ}$. The low sensitivity to bulge stars at negative longitudes, because of their large distance from us, may explain the difficulty in determining precisely the bulge orientation angle. Furthermore, it should be noted that these studies were based on integrated flux density, hence they were less sensitive to the distribution of stars along the line of sight than the present star count analysis.

\section{Conclusion}

We constructed a Monte Carlo fitting method to determine the spatial distribution of outer bulge and disc stars from comparisons between DENIS $K_{\mathrm{s}}$ and $J-K_{\mathrm{s}}$ stars counts and simulations from the Besançon model of the Galaxy. Our best parameters show a thin disc that has a central hole, as often seen in barred spiral galaxies, and a boxy prolate outer bulge with a major axis lying almost in the Galactic plane, as obtained by most of the other studies. However, our orientation angle (with respect to the Sun-center direction), $\phi=10.6^{\circ} \pm 3^{\circ}$, is slightly smaller than the mean value found in the literature.

The best fit among the different bulge ages which have been tested is the youngest: 7.9 Gyr. Future fits involving deeper data and more fields close to the Galactic plane are planned to test other luminosity functions, with younger ages and varying metallicities.

This study of the inner Galaxy is based on near infrared data, using low extinction windows. This approach does not allow us to have fields very close to the Galactic plane and center. The use of mid-infrared observations, like ISOGAL (Omont et al. 2003) or GLIMPSE (Benjamin et al. 2003), or deep near infrared surveys like WIRCAM at the CFHT, soon to be available, will allow us to reach these fields. It would be interesting to be able to include these fields, especially to study the population of the inner bulge.

The boxy prolate shape and a possible young bulge age are compatible with a bar structure, and may be explained by a reduced time scale for stellar formation. However this photometric study is not sufficient to settle the nature of the formation scenario of the outer bulge. Kinematical data would be needed to confirm the bar nature of the outer bulge, and thence its scenario of formation.

Acknowledgements. This study could not have been made whithout the previous work of Emmanuel Chereul, when he was post-doctoral fellow at the Geneva Observatory. The authors thank Guy Simon who was deeply involved in the DENIS batch reduction process, the whole DENIS staff and all people who observed and collected the data. We thank Mathias Schultheis who gave us his extinction maps, Edouard Oblak who helped us with the Monte Carlo fitting method, and Doug Marshall who corrected the english. Sébastien Picaud has benefited from an allowance from the Région de Franche-Comté.

\section{References}

Alard, C. 2001, A\&A, 379, L44

Alongi, M., Bertelli, G., Bressan, A., et al. 1993, A\&AS, 244, 95

Baggett, W. E., Baggett, S. M., \& Anderson, K. S. J. 1996, in Barred Galaxies, ed. R. Buta, D. Crocker, \& B. Elmegreen, IAU Coll., 157, ASP Conf. Ser., 91, 91

Benjamin, R. A., Churchwell, E., Babler, L., et al. 2003, PASP

Bertelli, G., Bressan, A., Chiosio, C., Fagotto, F., \& Nasi, E. 1994, A\&A, 301, 381

Bienaymé, O., Robin, A. C., \& Crézé, M. 1987, A\&A, 180, 94

Binney, J., Gerhard, O., Stark, A., Bally, J., \& Uchida, K. 1991, MNRAS, 252, 210

Binney, J., Gerhard, O., \& Spergel, D. 1997, MNRAS, 288, 365

Bissantz, N., \& Gerhard, O. 2002, MNRAS, 330, 591

Bissantz, N., Englmaier, P., \& Gerhard, O. E. 2003, MNRAS, 340, 949

Blitz, L., \& Spergel, D. N. 1991, ApJ, 379, 631

Bressan, A., Fagotto, F., Bertelli, G., \& Chiosi, C. 1993, A\&AS, 100, 647

Bruzual, G., Barbuy, B., Ortolani, S., et al. 1997, AJ, 114, 1531

Castelli, F., Graton, R. G., \& Kurucz, R. L. 1997, A\&A, 318, 841

Deguchi, S., Matsumoto, S., \& Wood, P. 1998, PASJ, 50, 597

Deguchi, S., Fujii, T., Nakashima, J., \& Wood, P. 2002, PASJ, 54, 719

Dwek, E., Arendt, R. G., Hauser, M. G., et al. 1995, ApJ, 445, 716

Einasto, J. 1979, The Large Scale Characteristics of the Galaxy, ed. W. B. Burton, IAU Symp., 84, 451

Epchtein, N., de Batz, B., Capoani, L., et al. 1997, The messenger, 87, 27

Fagotto, F., Bressan, A., Bertelli, G., \& Chiosi, C. 1994a, A\&AS, 104, 365 
Fagotto, F., Bressan, A., Bertelli, G., \& Chiosi, C. 1994b, A\&AS, 105, 29

Feast, M., \& Whitelock, P. 2000, MNRAS, 317, 460

Fouqué, P., Chevallier, L., Cohen, M., et al. 2000, A\&A, 141, 313

Freeman, K. C. 1970, ApJ, 160

Freudenreich, H. T. 1998, ApJ, 492, 495

Frogel, J. A., Tiede, G. P., \& Kuchinski, L. E. 1999, AJ, 117, 2296

Girardi, L., Bressan, A., Chiosi, C., Bertelli, G., \& Nasi, E. 1996, A\&AS, 117, 113

Girardi, L., Bertelli, G., Bressan, A., et al. 2002, A\&A, 391, 195

Haywood, M., Robin, A. C., \& Crézé, M. 1997, A\&A, 320, 440

Ibata, R., \& Gilmore, G. 1995, MNRAS, 275, 605

Kendall, M. G., \& Stuart, A. 1973a, The Advanced Theory of Statistics, Vol 2., Chap. 8, ed. Griffin (London)

Kendall, M. G., \& Stuart, A. 1973, The Advanced Theory of Statistics, Vol 2., Chap. 18, ed. Griffin (London)

Kent, S. M., Dame, T. M., \& Fazio, G. 1991, ApJ, 378, 131

Lejeune, T., Cuisinier, F., \& Buser, R. 1997, A\&AS, 125, 229

Lejeune, T., Cuisinier, F., \& Buser, R. 1998, A\&AS, 130, 65

Lépine, J. R. D., \& Leroy, P. 2000, MNRAS, 313, 263

López-Corredoira, M., Hammersley, P. L., Garzón, F., Simonneau, E., \& Mahoney, T. J. 2000, MNRAS, 313, 392

López-Corredoira, M., Hammersley, P. L., Garzón, F., et al. 2001, A\&A, 373, 139

López-Corredoira, M., Cabrera-Lavers, A., Gerhard, O. E., \& Garzón, F. 2004, A\&A, accepted

Mathis, J. S. 1990, ARA\&A, 31, 575

Nakada, Y., Onaka, T., Yamamura, I., et al. 1991, Nature, 353, 140

Nakai, N. 1992, PASJ, 44, L27

Nikolaev, S., \& Weinberg, M. 1997, ApJ, 487, 885
Oblak, E. 1983, Proc. Statistical Methods in Astronomy Symp., Strasbourg 13-16 Sep. 1983, ESA SP-201, 85 (SEE N8419196 09-889)

Ohta, K., Hamabe, M., \& Wakamatsu, K. 1990, ApJ, 357, 71

Omont, A., Gilmore, G. F., Alard, C., et al. 2003, A\&A, 403, 975

Picaud, S., Cabrera-Lavers, A., \& Garzón, F. 2003, A\&A, 408, 141

Reylé, C., \& Robin, A. C. 2001, A\&A, 373, 886

Reylé, C., \& Robin, A. C. 2002, A\&A, 384, 403

Reylé, C., Robin, A. C., Scholz, R.-D., \& Irwin, M. 2002, A\&A, 390, 491

Robin, A., \& Crézé, M. 1986, A\&A, 157, 71

Robin, A. C., Haywood, M., Crézé, M., Ojha, D. K., \& Bienaymé, O. 1996, A\&A, 305, 125

Robin, A. C., Reylé, C., \& Crézé, M. 2000, A\&A, 359, 103

Robin, A. C., Reylé, C., Derrière, S., \& Picaud, S. 2003, A\&A, 409, 523

Robin, A. C., Reylé, C., Derrière, S., \& Picaud, S. 2004, A\&A, 416, 157

Ruphy, S., Robin, A. C., Epchtein, N., et al. 1996, A\&A, 313, L21

Schultheis, M., Ganesh, S., Simon, G., et al. 1999, A\&A, 349, L69

Sevenster, M., Saha, P., Valls-Gabaud, D., \& Roger, F. 1999, MNRAS, 307, 584

Stanek, K. Z., Udalski, A., Szymanski, M., et al. 1997, ApJ, 477, 163

Weiland, J. L., Arendt, R. G., Berriman, G. B., et al. 1994, ApJ, 425, L81

Weinberg, M. 1992, ApJ, 420, 597

Weiner, B., \& Sellwood, J. A. 1999, ApJ, 524, 112

Zhao, H. S. 1996, MNRAS, 283, 149

Zhao, H. S., \& Mao, S. 1996, MNRAS, 283, 1197

Zoccali, M., Renzini, A., Ortolani, S, et al. 2003, A\&A, 399, 931 


\section{Online Material}




\section{Appendix A: Semi-Gaussian drawings}

At each iteration of the fitting program, Gaussian drawings determining the new $p$ points were made using different values of dispersion at the right and at the left of the median point on the eigenframe axes. The aim was to describe as fully as possible the morphology of the maximum likelihood region and reproduce it in the new drawings.

Let $\left(\xi^{k}, k=1, \ldots, m\right)$ be a family of $m$ points of the normalized 11D space of parameters. These points are sorted with respect to their reduced log-likelihood (see Appendix B), $L_{1}$ and $L_{m}$ being respectively the maximal and minimal likelihoods, and weighted, the weight $w_{k}$ being defined by:

$w_{k}=\exp \left(-1.5 \frac{L_{k}-L_{1}}{L_{m}-L_{1}}\right)$.

This formula allows us to enhance (under a certain limit) the contribution of best points in the following formulae.

Let $\bar{\xi}$ be the median of the $m$ weighted points and $\vec{V}_{j}$ be the eigenvector $j$ of their covariance matrix. Their values on the $i$ coordinate in the initial frame are respectively $\bar{\xi}_{i}$ and $V_{i j}$. We call $\hat{\xi}_{j}^{k}$ the coordinate $j$ of $\xi^{k}$ in the eigenframe $\left(\bar{\xi},\left\{\vec{V}_{j}, j=\right.\right.$ $1 \ldots 11\})$.

We define the semi-dispersion $\sigma_{-}$and $\sigma_{+}$by:

$\sigma_{-}=\sqrt{\sum_{\hat{\xi}_{j}^{k}<0} w_{j} \cdot\left(\hat{\xi}_{j}^{k}\right)^{2}}$ et $\sigma_{+}=\sqrt{\sum_{\hat{\xi}_{j}^{k} \geq 0} w_{j} \cdot\left(\hat{\xi}_{j}^{k}\right)^{2}}$.

Let $\left(\zeta_{j}^{l}, l=1 \ldots p, j=1 \ldots 11\right)$ be a family of $p \times 11$ coordinates obtained using a Gaussian drawing of mean 0 and dispersion 1 . The coordinates $\left(\tilde{\xi}_{i}^{l}, l=1, . ., p\right)$ in the initial frame of the $p$ new points to be tested are then deduced from the following formula:

$\tilde{\xi}_{i}^{l}=\bar{\xi}_{i}+\sigma_{-} \sum_{\zeta_{j<0}^{l}} V_{i j} \zeta_{i}^{l}+\sigma_{+} \sum_{\zeta_{j \geq 0}^{l}} V_{i j} \zeta_{i}^{l}$

\section{Appendix B: Likelihood and $\chi^{2}$ formulae}

Generally, when the agreement between observations and an analytic model is tested, only the data has Poisson noise, and usual likelihood or $\chi^{2}$ formulae are used. In the case of the Besançon model, initial simulations also have a Poisson noise. This particularity must be taken into account in the formulae of likelihood and $\chi^{2}$, as well as the fact that the model counts are deduced from initial simulations using weightings.

Let $i$ identify one of the bins. Correlations between adjacent bins are neglected. Let $y_{i}$ represent data star counts and $z_{i}$ be model counts. $y_{i}$ (integer) follows a Poisson law around $Y_{i}$ (unknown). $z_{i}$ (real) is obtained from an initial simulation star count $z_{i}=\alpha z_{0 i}, \alpha$ being the weight $t^{1}$ and $z_{0 i}$ (integer) following a Poisson law around $Z_{0 i}=\frac{1}{\alpha} Z_{i}\left(Z_{i}\right.$ and $Z_{0 i}$ being real $)$.

\section{B.1. Reduced log-likelihood}

Hereafter, probabilities (for discrete variables) will be noted $P$ and densities of probabilities (for continuous variables) will be noted $f$.

\footnotetext{
1 The case $\alpha=0$ is excluded.
}

By definition, the likelihood $\mathcal{L}_{i}$ (Kendall \& Stuart 1973) is the probability for an observed star count to take the value $y_{i}$, assuming that the model is correct, i.e. $Y_{i}=Z_{i}=\alpha Z_{0 i}$. One has:

$$
\mathcal{L}_{i}=P\left(y_{i} / z_{i}\right)=\int P\left(y_{i} / Z_{i}\right) f\left(Z_{i} / z_{i}\right) \mathrm{d} Z_{i}
$$

$y_{i}$ follows a Poisson law around $Z_{i}$, so:

$$
P\left(y_{i} / Z_{i}\right)=\frac{1}{y_{i} !} Z_{i}^{y_{i}} \mathrm{e}^{-Z_{i}} \text {. }
$$

One has: $f\left(Z_{i} / z_{i}\right)=\frac{1}{\alpha} f\left(Z_{0 i} / z_{0 i}\right)$. According to Bayes' theorem:

$$
f\left(Z_{0 i} / z_{0 i}\right)=\frac{P\left(z_{0 i} / Z_{0 i}\right) f\left(Z_{0 i}\right)}{\int P\left(z_{0 i} / Z_{0 i}\right) f\left(Z_{0 i}\right) \mathrm{d} Z_{0 i}} .
$$

Having no information a priori on $Z_{0 i}$, one can say that all $f\left(Z_{0 i}\right)$ are equal ${ }^{2}$ (at least around $z_{0 i}$, where $P\left(z_{0 i} / Z_{0 i}\right)$ is not negligible), so one can simplify Eq. (B.3):

$$
\begin{aligned}
& f\left(Z_{0 i} / z_{0 i}\right)=\frac{P\left(z_{0 i} / Z_{0 i}\right)}{\int P\left(z_{0 i} / Z_{0 i}\right) \mathrm{d} Z_{0 i}} \\
& P\left(z_{0 i} / Z_{0 i}\right)=\frac{1}{z_{0 i} !} Z_{0 i}{ }^{z_{0 i}} \mathrm{e}^{-Z_{0 i}}, \int P\left(z_{0 i} / Z_{0 i}\right) \mathrm{d} Z_{0 i}=1, \text { so: } \\
& f\left(Z_{i} / z_{i}\right)=\frac{1}{\alpha} \frac{1}{z_{0 i} !} Z_{0 i}{ }^{z_{0 i}} \mathrm{e}^{-Z_{0 i}} .
\end{aligned}
$$

On substituting for $P\left(y_{i} / Z_{i}\right)$ from Eq. (B.2), for $f\left(Z_{i} / z_{i}\right)$ from Eq. (B.4), putting these into Eq. (B.1) and replacing $Z_{i}$ by $\alpha Z_{0 i}$, one finds (with $\left.\zeta=(1+\alpha) Z_{0 i}\right)$ :

$$
\begin{aligned}
\mathcal{L}_{i} & =\int \frac{1}{y_{i} !}\left(\alpha Z_{0 i}\right)^{y_{i}} \mathrm{e}^{-\alpha Z_{0 i}} \frac{1}{\alpha} \frac{1}{z_{0 i} !} Z_{0 i}{ }^{z_{0 i}} \mathrm{e}^{-Z_{0 i}} \mathrm{~d}\left(\alpha Z_{0 i}\right) \\
& =\frac{1}{y_{i} ! z_{0 i} !} \frac{\alpha^{y_{i}}}{(1+\alpha)^{y_{i}+z_{0 i}+1}} \int \zeta^{y_{i}+z_{0 i}} \mathrm{e}^{-\zeta} \mathrm{d} \zeta \\
& =\frac{\left(y_{i}+z_{0 i}\right) !}{y_{i} ! z_{0 i} !} \frac{\alpha^{y_{i}}}{(1+\alpha)^{y_{i}+z_{0 i}+1}} \\
& =\frac{\left(y_{i}+z_{0 i}\right) !}{y_{i} ! z_{0 i} !} z_{0_{i}}^{z_{0 i}+1} \frac{z_{i}^{y_{i}}}{\left(z_{i}+z_{0 i}\right)^{y_{i}+z_{0 i}+1}} .
\end{aligned}
$$

Actually, we usually use the reduced log-likelihood, which is the natural logarithm of the likelihood minus the likelihood computed with $z_{i}$ replaced by $y_{i}$ :

$$
\begin{aligned}
L_{i} & =\ln \frac{\left(y_{i}+z_{0 i}\right) !}{y_{i} ! z_{0 i} !} z_{0_{i}}^{z_{0 i}+1} \frac{z_{i}^{y_{i}}}{\left(z_{i}+z_{0 i}\right)^{y_{i}+z_{0 i}+1}} \\
& -\ln \frac{\left(y_{i}+z_{0 i}\right) !}{y_{i} ! z_{0 i} !} z_{0_{i}}^{z_{0 i}+1} \frac{y_{i}^{y_{i}}}{\left(y_{i}+z_{0 i}\right)^{y_{i}+z_{0 i}+1}} \\
& =y_{i} \ln \left(\frac{z_{i}}{y_{i}}\right)-\left(y_{i}+z_{0 i}+1\right) \ln \left(\frac{z_{0 i}+z_{i}}{z_{0 i}+y_{i}}\right) .
\end{aligned}
$$

Finally, by summing all the bins, one obtains:

$L=\sum_{i}\left[y_{i} \ln \left(\frac{z_{i}}{y_{i}}\right)-\left(y_{i}+z_{0 i}+1\right) \ln \left(\frac{z_{0 i}+z_{i}}{z_{0 i}+y_{i}}\right)\right]$

The relative difference between the reduced log-likelihood obtained here and the usual one (i.e. without model noise: $L_{0}=$ $\sum_{i}\left[y_{i} \ln \left(\frac{z_{i}}{y_{i}}\right)-\left(z_{i}-y_{i}\right)\right)$ varies between $8 \%$ and $12 \%$, which is not negligible.

\footnotetext{
2 Bayes' Postulate.
} 
S. Picaud and A. C. Robin: The shape of the Galactic bulge from DENIS, Online Material $p 3$

Table 4. Results of the second round of fits using the Pad7.9 luminosity function. The table is composed of 3 subtables, one for each density profile $(E G$ and $S$ ). Each subtable is formed by two couples of lines giving the median $(\mu)$ and dispersion $(\sigma)$ of the values. Each pair of lines corresponds to a group of solutions (see text): fits with $\phi \geq 4.5^{\circ}$ (upper) and fits with $\phi<4.5^{\circ}$ (lower). The number of fits of each group is given at the beginning of the first line. $L$ and $\chi_{r}$ mean global log-reduced likelihood and square root of $\chi^{2}$ per bin, respectively (see Appendix B for more explanations).

\begin{tabular}{|c|c|c|c|c|c|c|c|c|c|c|c|c|c|c|c|}
\hline & pad7.9 & & $\begin{array}{l}\phi \\
\circ\end{array}$ & $\begin{array}{l}\beta \\
\beta\end{array}$ & $\begin{array}{r}x_{0} \\
\mathrm{kpc}\end{array}$ & $\begin{array}{c}y_{0} \\
\mathrm{kpc}\end{array}$ & $\begin{array}{r}z_{0} \\
\mathrm{kpc}\end{array}$ & $\begin{array}{c}\rho_{0} \\
\star \cdot \mathrm{pc}^{-3}\end{array}$ & $\begin{array}{c}R_{\mathrm{c}} \\
\mathrm{kpc}\end{array}$ & $\begin{array}{c}R_{\mathrm{d}} \\
\mathrm{kpc}\end{array}$ & $\begin{array}{r}R_{\mathrm{h}} \\
\mathrm{kpc}\end{array}$ & $C_{\|}$ & $C_{\perp}$ & $L$ & $\chi_{r}$ \\
\hline \multirow{4}{*}{$\mathrm{E}$} & 16 & $\mu_{i}$ & 7.1 & 0.6 & 1.35 & 0.41 & 0.32 & 18.62 & 3.66 & 2.44 & 1.25 & 2.969 & 2.804 & -1799 & 1.79 \\
\hline & & $\sigma_{i}$ & 2.4 & 1.1 & 0.25 & 0.08 & 0.02 & 2.42 & 1.04 & 0.10 & 0.11 & 0.699 & 1.146 & 43 & 0.02 \\
\hline & 4 & $\mu_{i}$ & 2.6 & 0.0 & 1.73 & 0.56 & 0.32 & 16.56 & 2.23 & 2.46 & 1.24 & 3.392 & 1.112 & -1769 & 1.77 \\
\hline & & $\sigma_{i}$ & 0.3 & 0.5 & 0.09 & 0.03 & 0.02 & 0.48 & 0.23 & 0.14 & 0.13 & 0.630 & 0.090 & 20 & 0.01 \\
\hline \multirow{4}{*}{$\mathrm{G}$} & 17 & $\mu_{i}$ & 10.6 & 0.4 & 1.60 & 0.47 & 0.40 & 9.84 & 3.44 & 2.30 & 1.27 & 3.201 & 3.751 & -1872 & 1.82 \\
\hline & & $\sigma_{i}$ & 3.8 & 0.9 & 0.18 & 0.06 & 0.05 & 1.55 & 0.53 & 0.13 & 0.16 & 0.759 & 1.085 & 69 & 0.04 \\
\hline & 3 & $\mu_{i}$ & 2.0 & 0.3 & 2.16 & 0.78 & 0.39 & 8.78 & 2.78 & 2.46 & 1.31 & 2.675 & 1.021 & -1752 & 1.76 \\
\hline & & $\sigma_{i}$ & 0.3 & 0.9 & 0.39 & 0.10 & 0.01 & 1.31 & 0.20 & 0.04 & 0.17 & 0.914 & 0.250 & 111 & 0.05 \\
\hline \multirow[b]{2}{*}{ S } & 20 & $\mu_{i}$ & 10.6 & 0.8 & 1.82 & 0.53 & 0.45 & 11.48 & 3.71 & 2.35 & 1.31 & 3.375 & 3.489 & -1790 & 1.79 \\
\hline & 0 & $\sigma_{i}$ & 3.0 & 0.9 & 0.17 & 0.06 & 0.02 & 0.73 & 0.71 & 0.09 & 0.09 & 0.659 & 1.028 & 19 & 0.01 \\
\hline
\end{tabular}

Table 5. Results of the second round of fits using the Pad10 luminosity function.

\begin{tabular}{|c|c|c|c|c|c|c|c|c|c|c|c|c|c|c|c|}
\hline & pad10 & & $\begin{array}{l}\phi \\
\circ\end{array}$ & $\begin{array}{l}\beta \\
\circ\end{array}$ & $\begin{array}{c}x_{0} \\
\mathrm{kpc}\end{array}$ & $\begin{array}{c}y_{0} \\
\mathrm{kpc}\end{array}$ & $\begin{array}{c}z_{0} \\
\mathrm{kpc}\end{array}$ & $\begin{array}{c}\rho_{0} \\
\star \cdot \mathrm{pc}^{-3}\end{array}$ & $\begin{array}{c}R_{\mathrm{c}} \\
\mathrm{kpc}\end{array}$ & $\begin{array}{c}R_{\mathrm{d}} \\
\mathrm{kpc}\end{array}$ & $\begin{array}{c}R_{\mathrm{h}} \\
\mathrm{kpc}\end{array}$ & $C_{\|}$ & $C_{\perp}$ & $L$ & $\chi_{r}$ \\
\hline \multirow{4}{*}{$\mathrm{E}$} & 9 & $\mu_{i}$ & 5.4 & 0.0 & 1.54 & 0.42 & 0.32 & 18.92 & 3.35 & 2.33 & 1.30 & 2.833 & 2.112 & -1921 & 1.85 \\
\hline & & $\sigma_{i}$ & 1.9 & 0.5 & 0.11 & 0.04 & 0.06 & 1.50 & 0.73 & 0.14 & 0.18 & 0.788 & 1.514 & 54 & 0.03 \\
\hline & 11 & $\mu_{i}$ & 2.6 & 0.5 & 1.79 & 0.54 & 0.31 & 18.78 & 2.54 & 2.38 & 1.30 & 3.016 & 1.044 & -1862 & 1.82 \\
\hline & & $\sigma_{i}$ & 0.3 & 0.7 & 0.11 & 0.05 & 0.02 & 1.25 & 0.34 & 0.11 & 0.17 & 0.776 & 0.212 & 30 & 0.02 \\
\hline \multirow{4}{*}{$\mathrm{G}$} & 8 & $\mu_{i}$ & 5.4 & 0.2 & 1.93 & 0.51 & 0.39 & 9.69 & 3.68 & 2.33 & 1.18 & 2.640 & 2.451 & -1957 & 1.87 \\
\hline & & $\sigma_{i}$ & 2.4 & 0.5 & 0.22 & 0.05 & 0.02 & 0.93 & 0.84 & 0.18 & 0.20 & 0.689 & 1.022 & 61 & 0.03 \\
\hline & 12 & $\mu_{i}$ & 2.0 & 0.2 & 2.19 & 0.62 & 0.39 & 9.22 & 2.76 & 2.51 & 1.09 & 2.666 & 1.373 & -1886 & 1.83 \\
\hline & & $\sigma_{i}$ & 0.7 & 0.5 & 0.12 & 0.07 & 0.02 & 0.37 & 0.98 & 0.12 & 0.19 & 0.564 & 0.289 & 51 & 0.03 \\
\hline \multirow{4}{*}{$S$} & 17 & $\mu_{i}$ & 8.6 & 0.1 & 2.07 & 0.54 & 0.47 & 11.45 & 3.11 & 2.40 & 1.19 & 2.839 & 3.521 & -1934 & 1.86 \\
\hline & & $\sigma_{i}$ & 2.0 & 0.6 & 0.18 & 0.04 & 0.02 & 0.77 & 0.74 & 0.10 & 0.12 & 0.836 & 0.803 & 33 & 0.01 \\
\hline & 3 & $\mu_{i}$ & 3.1 & -0.1 & 2.35 & 0.69 & 0.45 & 11.06 & 2.23 & 2.51 & 1.12 & 3.646 & 1.545 & -1900 & 1.83 \\
\hline & & $\sigma_{i}$ & 0.2 & 1.1 & 0.09 & 0.04 & 0.02 & 0.77 & 0.21 & 0.06 & 0.11 & 0.486 & 0.250 & 12 & 0.01 \\
\hline
\end{tabular}

Table 6. Results of the second round of fits using the Pad12.6 luminosity function.

\begin{tabular}{|c|c|c|c|c|c|c|c|c|c|c|c|c|c|c|c|}
\hline & pad12.6 & & $\begin{array}{l}\phi \\
\end{array}$ & $\begin{array}{l}\beta \\
\circ\end{array}$ & $\begin{array}{c}x_{0} \\
\mathrm{kpc}\end{array}$ & $\begin{array}{c}y_{0} \\
\mathrm{kpc}\end{array}$ & $\begin{array}{c}z_{0} \\
\mathrm{kpc}\end{array}$ & $\begin{array}{c}\rho_{0} \\
\star \cdot \mathrm{pc}^{-3}\end{array}$ & $\begin{array}{c}R_{\mathrm{c}} \\
\mathrm{kpc}\end{array}$ & $\begin{array}{c}R_{\mathrm{d}} \\
\mathrm{kpc}\end{array}$ & $\begin{array}{l}R_{\mathrm{h}} \\
\mathrm{kpc}\end{array}$ & $\overline{C_{\|}}$ & $C_{\perp}$ & $L$ & $\chi_{r}$ \\
\hline \multirow{4}{*}{ E } & 11 & $\overline{\mu_{i}}$ & 4.5 & 0.3 & 1.74 & 0.41 & 0.33 & 18.01 & 3.39 & 2.42 & 1.13 & 2.380 & 1.959 & -2151 & 1.95 \\
\hline & & $\sigma_{i}$ & 0.8 & 0.6 & 0.22 & 0.04 & 0.03 & 2.40 & 0.61 & 0.08 & 0.09 & 0.701 & 1.145 & 53 & 0.03 \\
\hline & 9 & $\mu_{i}$ & 2.5 & 0.4 & 1.92 & 0.46 & 0.31 & 18.32 & 2.86 & 2.48 & 1.22 & 2.597 & 1.375 & -2085 & 1.92 \\
\hline & & $\sigma_{i}$ & 0.5 & 0.3 & 0.20 & 0.04 & 0.02 & 1.72 & 0.39 & 0.14 & 0.19 & 0.516 & 0.154 & 33 & 0.02 \\
\hline \multirow{4}{*}{$\mathrm{G}$} & 11 & $\mu_{i}$ & 6.9 & -0.5 & 2.07 & 0.49 & 0.41 & 9.97 & 3.27 & 2.37 & 1.17 & 2.570 & 3.299 & -2200 & 1.97 \\
\hline & & $\sigma_{i}$ & 2.8 & 0.8 & 0.29 & 0.05 & 0.07 & 1.72 & 0.44 & 0.10 & 0.13 & 0.783 & 0.927 & 115 & 0.05 \\
\hline & 9 & $\mu_{i}$ & 1.8 & 0.1 & 2.36 & 0.64 & 0.39 & 9.27 & 2.44 & 2.55 & 1.02 & 3.079 & 1.204 & -2106 & 1.93 \\
\hline & & $\sigma_{i}$ & 0.5 & 1.0 & 0.13 & 0.05 & 0.03 & 0.61 & 0.61 & 0.09 & 0.13 & 0.783 & 0.295 & 51 & 0.03 \\
\hline \multirow{4}{*}{$S$} & 10 & $\mu_{i}$ & 7.9 & -0.4 & 2.22 & 0.53 & 0.45 & 11.39 & 3.15 & 2.39 & 1.16 & 3.367 & 3.618 & -2181 & 1.97 \\
\hline & & $\sigma_{i}$ & 2.0 & 0.9 & 0.21 & 0.04 & 0.02 & 0.41 & 0.66 & 0.06 & 0.08 & 0.646 & 1.115 & 40 & 0.02 \\
\hline & 10 & $\mu_{i}$ & 2.6 & 0.4 & 2.61 & 0.67 & 0.46 & 10.76 & 3.04 & 2.41 & 1.16 & 3.082 & 1.426 & -2106 & 1.93 \\
\hline & & $\sigma_{i}$ & 0.5 & 1.1 & 0.21 & 0.07 & 0.03 & 0.58 & 0.82 & 0.08 & 0.10 & 0.533 & 0.240 & 54 & 0.02 \\
\hline
\end{tabular}

B.2. $\chi_{r}$

$y_{i}$ is a Poisson random variable with an average and variance $Y_{i}$. This variance is unknown and its only approximation available is the value $y_{i}$. $z_{i}$ is a random variable with an average $\alpha Z_{0 i}$ and a variance $\alpha^{2} Z_{0 i}=\frac{Z_{i}^{2}}{Z_{0 i}}$. This variance is unknown and its only approximation available is the value $\frac{z_{i}^{2}}{z_{0 i}}$. 
S. Picaud and A. C. Robin: The shape of the Galactic bulge from DENIS, Online Material p 4

Table 7. Results of the second round of fits using the BC10 luminosity function.

\begin{tabular}{|c|c|c|c|c|c|c|c|c|c|c|c|c|c|c|c|}
\hline & $\overline{\mathrm{BC} 10}$ & & $\begin{array}{l}\phi \\
\circ\end{array}$ & $\begin{array}{l}\beta \\
\beta \\
\circ\end{array}$ & $\begin{array}{c}x_{0} \\
\mathrm{kpc}\end{array}$ & $\begin{array}{c}y_{0} \\
\mathrm{kpc}\end{array}$ & $\begin{array}{c}z_{0} \\
\mathrm{kpc}\end{array}$ & $\begin{array}{c}\rho_{0} \\
\star \cdot \mathrm{pc}^{-3}\end{array}$ & $\begin{array}{c}R_{\mathrm{c}} \\
\mathrm{kpc}\end{array}$ & $\begin{array}{c}R_{\mathrm{d}} \\
\mathrm{kpc}\end{array}$ & $\begin{array}{c}R_{\mathrm{h}} \\
\mathrm{kpc}\end{array}$ & 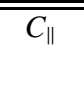 & $\overline{C_{\perp}}$ & $\overline{L L}$ & $\overline{\chi_{r}}$ \\
\hline \multirow{4}{*}{$\mathrm{E}$} & 17 & $\mu_{i}$ & 8.5 & 0.7 & 1.26 & 0.46 & 0.35 & 21.39 & 2.38 & 2.45 & 1.28 & 3.235 & 3.715 & -2048 & 1.92 \\
\hline & & $\sigma_{i}$ & 2.3 & 1.3 & 0.14 & 0.06 & 0.03 & 1.63 & 0.36 & 0.11 & 0.13 & 0.771 & 1.206 & 26 & 0.01 \\
\hline & 3 & $\mu_{i}$ & 3.3 & -0.2 & 1.31 & 0.58 & 0.33 & 22.19 & 2.38 & 2.47 & 1.27 & 3.424 & 1.348 & -2031 & 1.92 \\
\hline & & $\sigma_{i}$ & 0.3 & 0.7 & 0.18 & 0.08 & 0.01 & 1.63 & 0.42 & 0.19 & 0.28 & 0.331 & 0.228 & 18 & 0.01 \\
\hline \multirow[b]{2}{*}{ G } & 20 & $\mu_{i}$ & 12.1 & 1.3 & 1.36 & 0.50 & 0.39 & 12.93 & 3.56 & 2.47 & 1.25 & 3.340 & 3.817 & -2102 & 1.94 \\
\hline & 0 & $\sigma_{i}$ & 1.7 & 1.2 & 0.10 & 0.02 & 0.02 & 0.82 & 0.78 & 0.12 & 0.15 & 0.762 & 0.351 & 21 & 0.01 \\
\hline \multirow[b]{2}{*}{$S$} & 20 & $\mu_{i}$ & 12.4 & 1.6 & 1.55 & 0.57 & 0.44 & 14.91 & 4.00 & 2.52 & 1.27 & 3.801 & 4.149 & -2027 & 1.92 \\
\hline & 0 & $\sigma_{i}$ & 1.5 & 0.7 & 0.06 & 0.03 & 0.01 & 0.48 & 0.63 & 0.10 & 0.14 & 0.423 & 0.704 & 14 & 0.01 \\
\hline
\end{tabular}

Table 8. Results of the second round of fits using the BC12 luminosity function.

\begin{tabular}{|c|c|c|c|c|c|c|c|c|c|c|c|c|c|c|c|}
\hline & BC12 & & $\begin{array}{l}\phi \\
\circ\end{array}$ & $\begin{array}{l}\beta \\
\circ\end{array}$ & $\begin{array}{c}x_{0} \\
\mathrm{kpc}\end{array}$ & $\begin{array}{c}y_{0} \\
\mathrm{kpc}\end{array}$ & $\begin{array}{c}z_{0} \\
\mathrm{kpc}\end{array}$ & $\begin{array}{c}\rho_{0} \\
\star \cdot \mathrm{pc}^{-3}\end{array}$ & $\begin{array}{c}R_{\mathrm{c}} \\
\mathrm{kpc}\end{array}$ & $\begin{array}{c}R_{\mathrm{d}} \\
\mathrm{kpc}\end{array}$ & $\begin{array}{c}R_{\mathrm{h}} \\
\mathrm{kpc}\end{array}$ & $C_{\|}$ & $C_{\perp}$ & $L$ & $\chi_{r}$ \\
\hline \multirow{4}{*}{ E } & 18 & $\mu_{i}$ & 8.0 & -0.8 & 1.40 & 0.46 & 0.36 & 21.56 & 2.24 & 2.44 & 1.22 & 3.607 & 3.257 & -1978 & 1.88 \\
\hline & & $\sigma_{i}$ & 1.2 & 1.8 & 0.13 & 0.04 & 0.03 & 1.84 & 0.42 & 0.12 & 0.16 & 0.594 & 0.666 & 36 & 0.02 \\
\hline & 2 & $\mu_{i}$ & 3.5 & 1.1 & 1.49 & 0.61 & 0.33 & 22.80 & 1.96 & 2.34 & 1.33 & 4.168 & 1.173 & -1936 & 1.87 \\
\hline & & $\sigma_{i}$ & 0.2 & 0.3 & 0.03 & 0.02 & 0.00 & 0.25 & 0.07 & 0.03 & 0.03 & 0.072 & 0.069 & 1 & 0.00 \\
\hline \multirow{4}{*}{ G } & 19 & $\mu_{i}$ & 11.8 & 0.6 & 1.41 & 0.48 & 0.37 & 14.26 & 3.49 & 2.37 & 1.28 & 4.080 & 4.163 & -1954 & 1.87 \\
\hline & & $\sigma_{i}$ & 2.3 & 0.7 & 0.08 & 0.04 & 0.02 & 0.66 & 0.85 & 0.11 & 0.11 & 0.741 & 0.896 & 22 & 0.01 \\
\hline & 1 & $\mu_{i}$ & 2.7 & 0.5 & 1.61 & 0.73 & 0.38 & 14.09 & 1.99 & 2.31 & 1.41 & 3.352 & 1.154 & -1958 & 1.88 \\
\hline & & $\sigma_{i}$ & 0.1 & 0.1 & 0.01 & 0.01 & 0.01 & 0.12 & 0.03 & 0.01 & 0.01 & 0.022 & 0.015 & 1 & 0.00 \\
\hline \multirow[b]{2}{*}{$S$} & 20 & $\mu_{i}$ & 12.3 & 0.9 & 1.58 & 0.55 & 0.45 & 16.79 & 4.01 & 2.35 & 1.36 & 3.654 & 4.106 & -1908 & 1.86 \\
\hline & 0 & $\sigma_{i}$ & 1.2 & 0.5 & 0.06 & 0.02 & 0.01 & 0.62 & 0.51 & 0.12 & 0.14 & 0.496 & 0.581 & 19 & 0.01 \\
\hline
\end{tabular}

So, $y_{i}-z_{i}$ is a variable with a variance $\sigma_{i}^{2} \approx y_{i}+\frac{z_{i}^{2}}{z_{0 i}}$. One therefore obtains the following formula of normalized residuals:

$$
r_{i}=\frac{y_{i}-z_{i}}{\sqrt{y_{i}+\frac{z_{i}^{2}}{z_{0 i}}}} .
$$

At the end, by summing over all the color magnitude bins of a window, one obtains the $\chi^{2}$ per field (or group of fields):

$\chi_{\mathrm{f}}^{2}=\sum_{i} r_{i}^{2}=\sum_{i} \frac{\left(y_{i}-z_{i}\right)^{2}}{y_{i}+\frac{z_{i}^{2}}{z_{0 i}}}$.

However, we would rather use the square root of the $\chi^{2}$ per bin $\chi_{\mathrm{rf}}=\sqrt{\frac{\chi^{2}}{n}}$ (with $n=$ number of bins) wich corresponds to the mean dispersion needed for random variables centered on the $y_{i}$ and used as model counts to obtain on average the same value of $\chi_{\mathrm{f}}^{2}$.

By summing over all the fields or groups of fields $\left(n_{\mathrm{f}}=88\right.$ in total), one obtains the global $\chi^{2}$ and the square root of the $\chi$ :

$\chi_{\mathrm{r}}=\frac{1}{n_{\mathrm{f}}} \sum_{f} \chi_{\mathrm{rf}}^{2}$

\section{B.3. Reduced log-likelihoods vs. $\chi_{r}$}

If the likelihood has been preferred to $\chi_{\mathrm{r}}$ to extract best sets of parameters in the fitting method, the $\chi_{\mathrm{r}}$ is a more practical tool

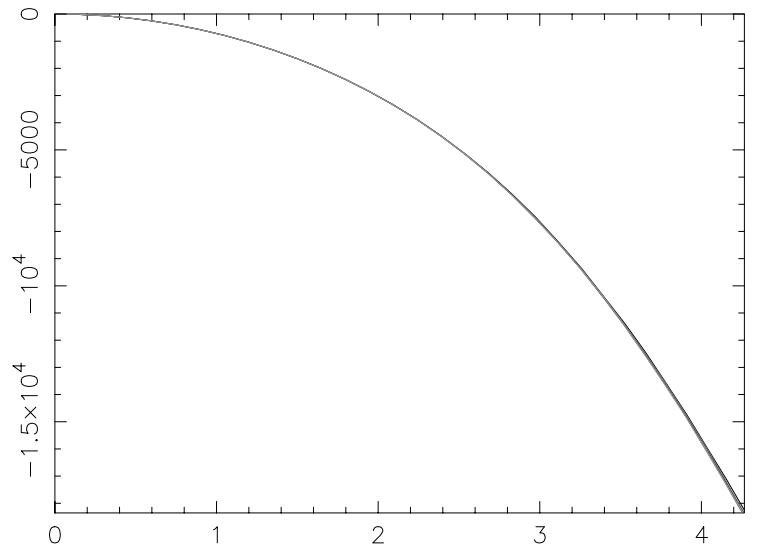

Fig. B.1. Reduced log-likelihoods (ordinates) versus square root of $\chi^{2}$ per bin.

than likelihood to "read" the quality of a fit, because it gives directly, in number of sigma, the mean distance between model and data. That is why the values of $\chi_{\mathrm{r}}$ have been calculated as well as likelihoods.

Figure B.1 presents the values of reduced log-likelihoods $L$ versus the square root of $\chi^{2}$ per bin $\chi_{r}$ obtained by random drawings. 